\title{
Random strict partitions and random shifted tableaux
}

\author{
Sho Matsumoto ${ }^{1} \cdot$ Piotr Śniady $^{2}$ (I)
}

Published online: 25 January 2020

(c) The Author(s) 2020

\begin{abstract}
We study asymptotics of random shifted Young diagrams which correspond to a given sequence of reducible projective representations of the symmetric groups. We show limit results (Law of Large Numbers and Central Limit Theorem) for their shapes, provided that the representation character ratios and their cumulants converge to zero at some prescribed speed. Our class of examples includes uniformly random shifted standard tableaux with prescribed shape as well as shifted tableaux generated by some natural combinatorial algorithms (such as shifted Robinson-Schensted-Knuth correspondence) applied to a random input.
\end{abstract}

Keywords Projective representations of the symmetric groups $\cdot$ Random strict partitions $\cdot$ Random shifted tableaux $\cdot$ Limit shape $\cdot$ Kerov's CLT $\cdot$ Shifted Schur-Weyl measure

Mathematics Subject Classification Primary 20C25; Secondary 20C30 - 60F05 . $05 \mathrm{E} 10$

\section{Introduction}

\subsection{Outlook}

This paper is arranged in the following way which is intended to make the learning curve somewhat less steep.

Dedidated to Etsuko Hirai and Takeshi Hirai.

$凶 \quad$ Piotr Śniady

psniady@impan.pl

https://psniady.impan.pl

Sho Matsumoto

shom@sci.kagoshima-u.ac.jp

1 Graduate School of Science and Engineering, Kagoshima University, 1-21-35, Korimoto, Kagoshima, Japan

2 Institute of Mathematics, Polish Academy of Sciences, ul. Śniadeckich 8, 00-656 Warszawa, Poland 
We start with Sects. 1.2-1.5 of this Introduction where we present basic notations related to strict partitions. We continue in Sects. 1.6-1.7 with two concrete example models which we aim to treat, namely random strict tableaux of prescribed shape and asymptotics of shifted Robinson-Schensted-Knuth correspondence.

These two example models turn out to be special cases of a more general and more abstract theory which we present later in the paper in Sect. 2. This general theory concerns random shifted Young diagrams related to reducible spin representations of the symmetric groups. We also state there the main results of the current paper, Theorems 2.5 and 2.6.

These general results are direct analogues of their classical counterparts for linear representations and usual (non-shifted) Young diagrams. Our strategy will be twofold: to revisit the ideas from the work of the second-named author [41] which concern the linear representations of the symmetric groups, as well as to use the link between the linear and the spin setup which we explored only recently [31].

Our reuse of the notion of the approximate factorization property for the character ratios (which appears in the assumptions of Theorems 2.5 and 2.6) has some novelty: in Sect. 3 we discuss this established notion with a new, more abstract and hopefully more elegant viewpoint of the category theory. This new approach seems applicable in a quite wide spectrum of setups, including the classical one [41].

The remaining part of the paper is tailored specifically for the needs of the setup of the asymptotic spin representation theory of the symmetric groups.

In Sect. 4 we present our key technical tools: Kerov-Olshanski algebra and its spin analogue. We prove the main technical difficulty of the current paper, Theorem 4.2.

In Sect. 5 we prove Theorem 5.1 which provides several equivalent, convenient characterizations for approximate factorization property for character ratios. This result directly implies the main results of the current paper, Theorems 2.5 and 2.6.

Section 6 is devoted to applications of the aforementioned Theorem 5.1. We construct a large collection of examples of sequences of representations with approximate factorization property for which the results of the current paper are applicable. In particular, we explain how Theorems 1.1, 1.3, Corollaries 1.4, 1.5 fit into the general framework of the approximate factorization property.

In Sect. 7 we recall the methods for finding explicitly the limit shape of (shifted) Young diagrams.

In "Appendix A" we recall some basic facts from the spin representation theory of the symmetric groups.

\subsection{Strict partitions}

Random partitions occur in mathematics and physics in a wide variety of contexts, in particular in the Gromov-Witten and Seiberg-Witten theories [32,47]. In the current paper we focus attention on a special class, namely on random strict partitions.

We recall that

$$
\xi=\left(\xi_{1}, \ldots, \xi_{\ell}\right)
$$



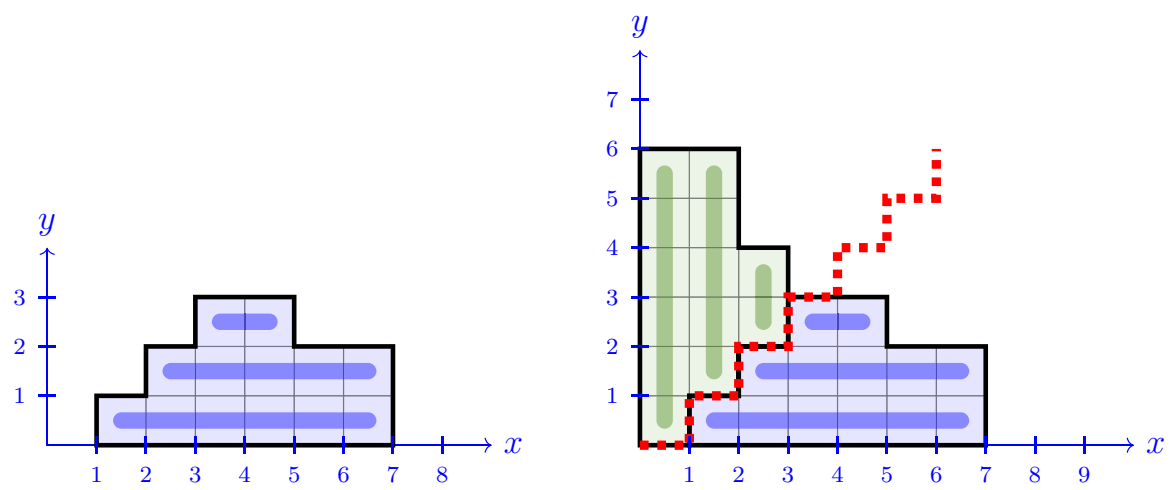

Fig. 1 Strict partition $\xi=(6,5,2)$ shown as a shifted Young diagram and its double $D(\xi)=$ $(7,7,5,3,2,2)$, cf. Sect. 4.3 .1

is a strict partition of an integer $n$ if $\xi_{1}>\cdots>\xi_{\ell}$ form a strictly decreasing sequence of positive integers such that $n=|\xi|=\xi_{1}+\cdots+\xi_{\ell}$, cf. [27, p. 9]. We denote by $\mathcal{S P}{ }_{n}$ the set of strict partitions of a given integer $n$ and by $\mathcal{S P}=\bigcup_{n \geq 0} \mathcal{S P}_{n}$ the set of all strict partitions.

It is convenient to represent graphically a strict partition $\xi$ by a shifted Young diagram, as it is shown in Fig. 1, which is a collection of boxes

$$
\left\{(x, y): x, y \in \mathbb{N}, 1 \leq y<x \leq y+\xi_{y}\right\}
$$

on the plane. We use the French notation for drawing diagrams as well as the usual Cartesian coordinate system $O X Y$ on the plane; in particular the variable $x$ indexes the columns and the variable $y$ indexes the rows. The rows and columns are indexed by natural numbers $\mathbb{N}=\{1,2, \ldots\}$.

Above and in the following it is convenient to view a strict partition (1) as an infinite sequence of non-negative integers

$$
\xi=\left(\xi_{1}, \ldots, \xi_{\ell}, 0,0, \ldots\right)
$$

by padding zeros at the end.

\subsection{Strict partitions: motivations and applications}

Strict partitions occur naturally in the context of spin representations of the symmetric groups $\mathfrak{S}_{n}$, cf. "Appendix A.2" later on, and in this article we shall concentrate on this link. Nevertheless, it is worth pointing out that they also appear in the theory of partially ordered sets as order filters in the root poset of type $B_{n}$, as well as they form an interesting infinite family of $d$-complete posets, which in turn is connected to fully commutative elements of some Coxeter groups [34,46]. Also, strict partitions are in a bijective correspondence with permutations which avoid patterns 132 and 312 [9]. 


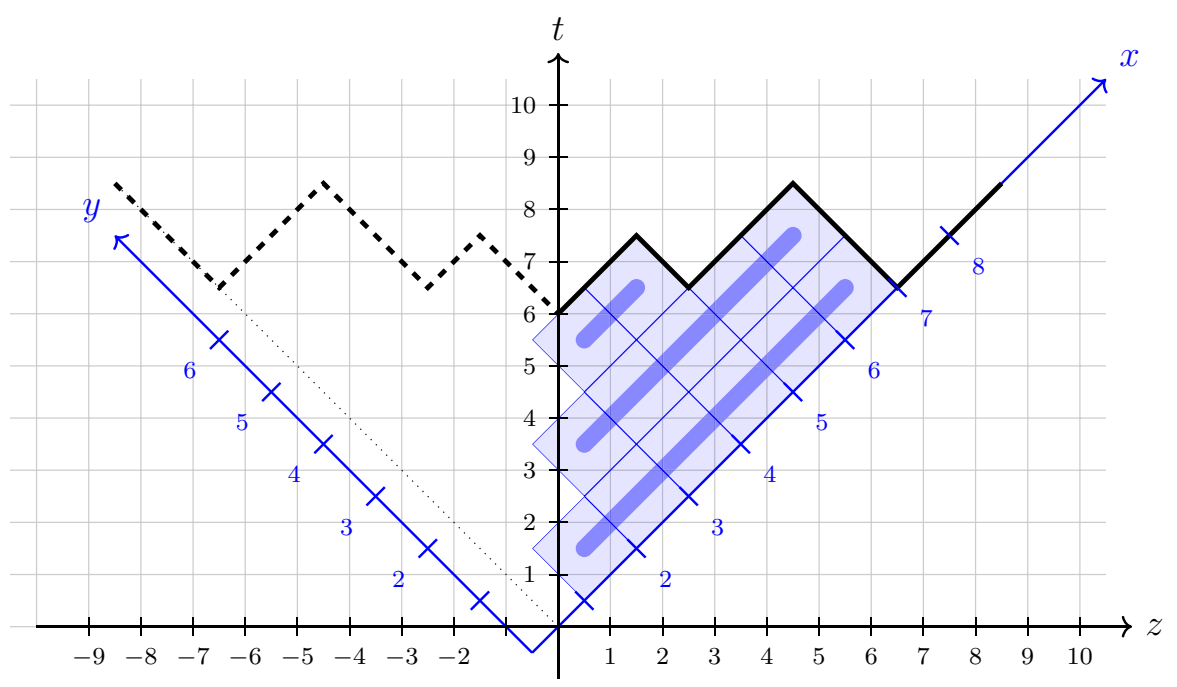

Fig. 2 The strict partition $\xi$ from Fig. 1 shown in the shifted Russian convention. The upper envelope of the boxes (the thick zig-zag line) is the graph of the profile $\omega \xi: \mathbb{R}_{+} \rightarrow \mathbb{R}_{+}$. If necessary, the domain of the profile $\omega_{\xi}: \mathbb{R} \rightarrow \mathbb{R}_{+}$can be extended to the whole real line (the thick dashed line)

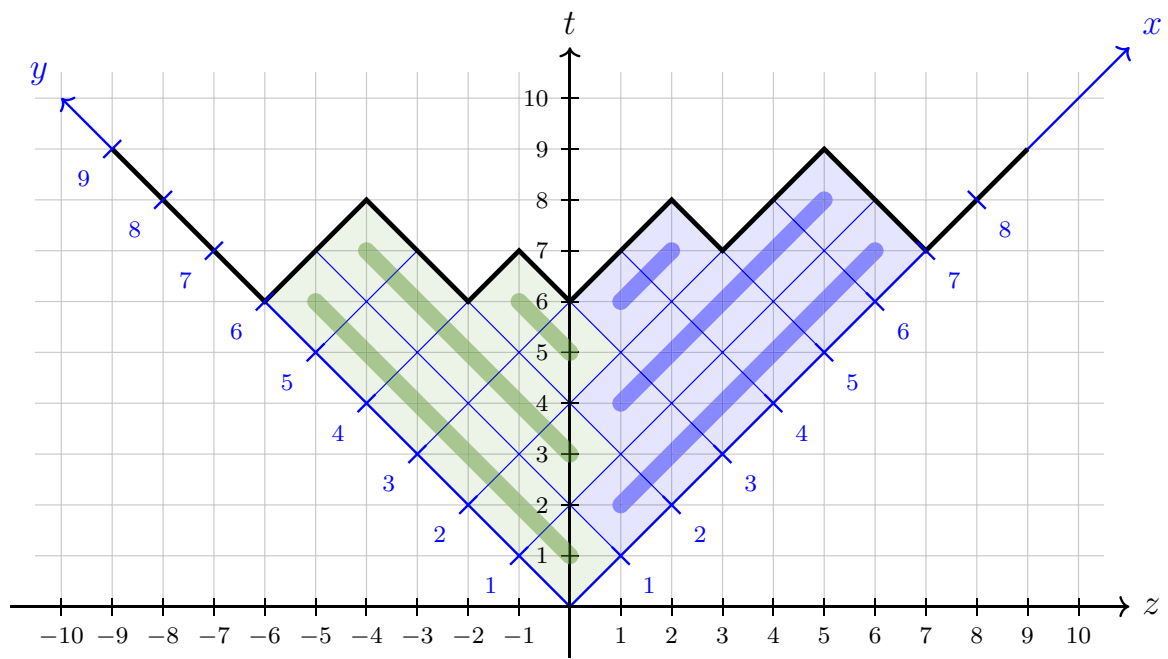

Fig. 3 The Young diagram $\lambda=D(\xi)$ from Fig. 1 shown in the Russian convention. The thick zig-zag line is the graph of the profile $\omega_{D(\xi)}: \mathbb{R} \rightarrow[0, \infty)$

\subsection{Drawing (strict) partitions and Young diagrams for asymptotic problems}

For asymptotic problems we need some way of drawing large strict partitions which would allow us to compare the shapes of such partitions with different numbers of boxes. In this section we shall present a convenient solution to this difficulty. 


\subsubsection{Shifted Russian convention for drawing shifted Young diagrams}

In the French convention we draw shifted Young diagrams on the plane using the Cartesian coordinate system $O X Y$, cf. Fig. 1, but for asymptotic questions it is convenient to draw them using the shifted Russian convention [10, Sect. 4.2.6] which corresponds to a new coordinate system $O Z T$ on the plane given by

$$
z=x-y-\frac{1}{2}, \quad t=x+y-\frac{1}{2},
$$

see Fig. 2. In this convention, the boundary of a shifted Young diagram $\xi$ (shown on Fig. 3 by the thick zigzag line), called its profile, is a graph of a function $\omega_{\xi}: \mathbb{R}_{+} \rightarrow \mathbb{R}_{+}$ on the positive half-line. If necessary, the domain of the profile can be extended to the whole real line by declaring that $\omega_{\xi}(-x)=\omega_{\xi}(x)$ for any $x \geq 0$. The graph of such a profile $\omega_{\xi}: \mathbb{R} \rightarrow \mathbb{R}_{+}$is shown on Fig. 2 as the union of the thick solid and the thick dashed lines.

\subsubsection{Russian convention for drawing Young diagrams}

In the French convention we draw the usual (non-shifted) Young diagrams on the plane using the Cartesian coordinate system $O X Y$, but for asymptotic questions it is convenient to draw them using the Russian convention (cf. Fig. 3) which corresponds to a new coordinate system $O Z T$ on the plane given by

$$
z=x-y, \quad t=x+y .
$$

In this convention, the boundary of a Young diagram $\lambda$ (shown on Fig. 3 by the thick zigzag line), called its profile is a graph of a function $\omega_{\lambda}: \mathbb{R} \rightarrow \mathbb{R}_{+}$.

\subsubsection{Continual Young diagrams: dilations of (shifted) Young diagrams}

We will say that a function $\omega: \mathbb{R} \rightarrow \mathbb{R}_{+}$is a continual Young diagram $[19,21]$ if

- $\left|\omega\left(z_{1}\right)-\omega\left(z_{2}\right)\right| \leq\left|z_{1}-z_{2}\right|$ for any $z_{1}, z_{2} \in \mathbb{R}$,

- $\omega(z)=|z|$ for sufficiently big values of $|z|$.

For a real number $r>0$ we can draw the boxes of the Young diagram $\lambda$ as squares of side $r$. Such an object—denoted $r \lambda$ and called dilated Young diagram —is usually no longer a Young diagram, but its profile $\omega_{r \lambda}$ is still well defined and is a continual Young diagram; note that

$$
\omega_{r \lambda}(z)=r \omega_{\lambda}\left(\frac{z}{r}\right) \quad \text { for any } z \in \mathbb{R}
$$

which can be viewed as an alternative definition of $\omega_{r \lambda}$.

For a shifted Young diagram $\xi$ the analogous operation of drawing boxes as squares of side $r>0$ and then looking on the profile of the resulting object is more delicate 
because we would have to adjust the additive terms in the linear transformations (3) to the new size of the boxes. For this reason we take the following analogue of (4):

$$
\omega_{r \xi}(z)=r \omega_{\xi}\left(\frac{z}{r}\right) \quad \text { for any } z \in \mathbb{R}
$$

as the definition of the profile of the dilated diagram $r \xi$.

\subsection{Shifted tableaux}

We recall that a shifted tableau is a filling of the boxes of a given shifted Young diagram $\xi$ which is weakly increasing along the rows and strictly increasing along the columns. Such a tableau is standard if each of the numbers $1,2, \ldots, n$ appears as an entry exactly once, where $n=|\xi|$ is the number of the boxes, cf. top of Fig. 4 for an example. For a shifted tableau $T$ of shape $\xi$ we denote by $T_{x, y}$ its entry in $x$-th column and $y$-th row for integers $x, y$ such that $1 \leq y<x \leq y+\xi_{y}$. For any integer $0 \leq i \leq n$ we denote by $T_{\leq i}=\left(\zeta_{1}, \zeta_{2}, \ldots\right) \in \mathcal{S} \mathcal{P}_{i}$ the strict partition which corresponds to the set of boxes of $T$ occupied by numbers which are $\leq i$; in other words

$$
\zeta_{y}=\#\left\{x: y<x \leq y+\xi_{y} \text { and } T_{x, y} \leq i\right\} .
$$

In the context of the representation theory of the symmetric groups, shifted tableaux play an analogous role (for spin representations) as the usual ("non-shifted") tableaux (for linear representations) and several classical combinatorial algorithms for tableaux have their shifted counterparts [36,39,45,49].

A special role is played by the staircase strict partition

$$
\Delta_{k}=(k, \ldots, 3,2,1)
$$

and by shifted tableaux with this shape; we call the latter staircase shifted tableaux. Such staircase shifted tableaux - apart from the aforementioned general context of the representation theory-appear in the combinatorics of the Coxeter groups; more specifically they are in a bijective correspondence with a natural class of objects which can be described in several equivalent ways:

- maximum length chains in the Tamari lattice [12],

- maximal chains in weak Bruhat order on 312-avoiding permutations in $\mathfrak{S}_{n}$ [12] which are also known under the name of 312-avoiding sorting networks,

- both 132- and 312-avoiding sorting networks [24, Proposition 3.12],

- the commutation class of the word

$$
\mathbf{w}_{0}=\left(s_{1} s_{2} \cdots s_{k-1}\right)\left(s_{1} s_{2} \cdots s_{k-2}\right) \cdots\left(s_{1} s_{2}\right)\left(s_{1}\right)
$$

in the symmetric group $\mathfrak{S}_{k}[38]$. 

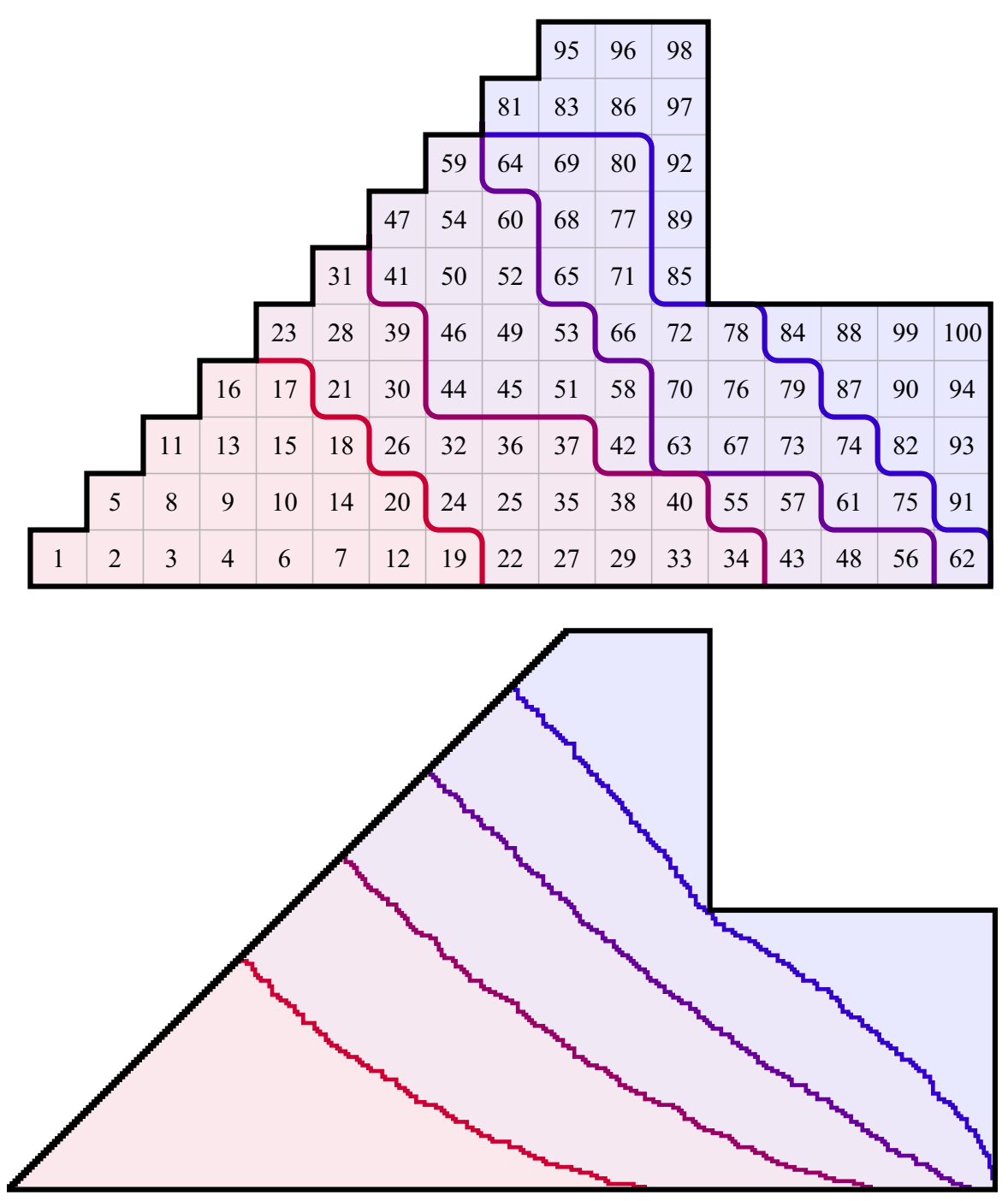

Fig. 4 Above: random shifted standard Young tableau with $n=100$ boxes, sampled with the uniform distribution on the set of shifted standard tableaux with fixed shape $\xi=(17,16, \ldots, 13,7,6, \ldots, 3)$. The coloured level curves indicate positions of $20 \%, 40 \%, 60 \%, 80 \%$ of the boxes with the smallest numbers. Below: analogous random tableau with $n=39600$ boxes. Individual boxes and the numbers filling the tableau are not shown

\subsection{The first example: random shifted standard tableaux with prescribed shape}

For $\xi \in \mathcal{S P}$ we denote by $\mathcal{T}_{\xi}$ the set of shifted standard tableaux with the presecribed shape $\xi$ and by $\mathbb{P}_{\xi}$ the uniform probability measure on $\mathcal{T}_{\xi}$. Such a uniformly random shifted tableau can be generated by the shifted hook walk algorithm [35] and is an important tool in some proofs of the hook length formula for the number $\left|\mathcal{T}_{\xi}\right|$ of shifted tableaux [35]. 


\subsubsection{Limit shape for random shifted tableaux}

Following the ideas of Pittel and Romik [33], a shifted tableau $T$ with shape $\xi \in \mathcal{S P}_{n}$ can be regarded as a three-dimensional stack of cubes over the two-dimensional shifted Young diagram $\xi$, with $T_{x, y}$ cubes stacked over the square $[x-1, x] \times[y-1, y] \times\{0\}$. Alternatively, the function $(x, y) \mapsto T_{\lceil x\rceil,\lceil y\rceil}$ can be interpreted as the graph of the (non-continuous) surface of the upper envelope of this stack.

It is convenient to rescale the unit boxes on the plane by the factor $\frac{1}{\sqrt{2 n}}$ in such a way that the area of $\xi$ becomes equal to $\frac{1}{2}$, and to rescale the height of the cubes by the factor $\frac{1}{n}$ in such a way that the heights of stacks of cubes are all between 0 and 1. In this way we may ask asymptotic questions about large random shifted tableaux using the language of random surfaces.

Before reading the exact form of the following result we recommend to consult the almost self-explanatory Fig. 4.

The following result states a kind of Law of Large Numbers result that if the (scaled down) shapes of the strict partitions $\left(\xi^{(k)}\right)$ converge to some limit shape $\Lambda$ then the aforementioned random surface which corresponds to a uniformly random shifted tableau $T^{(k)} \in \mathcal{T}_{\xi(k)}$ converges in probability towards some deterministic surface $F: \Lambda \rightarrow[0,1]$ in the sense of level curves. The latter sense of convergence means that the (scaled down) region on the plane occupied by the boxes with (scaled) height bounded from above by any fixed real number $\alpha$-in the physical geography the boundary of such a region is a curve called the contour curve or the level curveconverges in probability to the region where the surface $F$ takes values which are bounded from above by the same level $\alpha$.

Theorem 1.1 (Law of Large Numbers for random shifted tableaux) For each $k \geq 1$ let $\xi^{(k)}=\left(\xi_{1}^{(k)}, \xi_{2}^{(k)}, \ldots\right) \in \mathcal{S} \mathcal{P}_{n_{k}}$ for some sequence $\left(n_{k}\right)$ of positive integers which tends to infinity. We assume that the sequence of rescaled profiles converges to some limit

$$
\Omega_{1}:=\lim _{k \rightarrow \infty} \omega \frac{1}{\sqrt{2 n_{k}}} \xi^{(k)}
$$

in the sense of pointwise convergence of functions on $\mathbb{R}_{+}$. We denote by

$$
\Lambda=\left\{(x, y): 0 \leq y \leq x \text { and } x+y<\Omega_{1}(x-y)\right\}
$$

this limit shape drawn in the French coordinate system.

We also assume that the sequence $\left(\xi^{(k)}\right)$ is $C$-balanced [4], i.e. the length of the first row satisfies the bound

$$
\xi_{1}^{(k)}<C \sqrt{n_{k}}
$$

for some constant $C>0$ and all integers $k \geq 1$.

Then there exists a function $F: \Lambda \rightarrow[0,1]$ and the corresponding a family of level curves (drawn in the Russian convention) indexed by $0<\alpha<1$, defined for $z \geq 0$ by 


$$
\Omega_{\alpha}(z)=\sup \{x+y:(x, y) \in \Lambda \text { and } x-y=z \text { and } F(x, y) \leq \alpha\}
$$

we use the convention that if the supremum above is taken over the empty set then $\Omega_{\alpha}(z)=|z|$.

We denote by $T^{(k)}$ a random standard Young tableau, sampled with the uniform distribution on $\mathcal{T}_{\xi(k)}$. Then for each $0<\alpha<1$ the (rescaled by the factor $\frac{1}{\sqrt{2 n_{k}}}$ ) profile of the shifted Young diagram $T_{\leq \alpha n_{k}}^{(k)}$ converges in probability to $\Omega_{\alpha}$. In other words, for each $\epsilon>0$

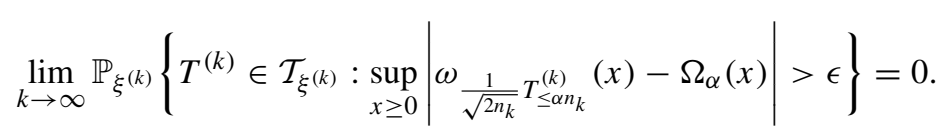

The proof and the exact form of the limit surface $F$ is postponed to Sect. 6.3. The results of the current paper can be also used to show that the fluctuations of the random surfaces $T^{(k)}$ around the limit shape are Gaussian.

Remark 1.2 With some minor effort, an analogous result for the usual (non-shifted) tableaux can be extracted from the work of Biane [4, Theorem 1.5.1]. This non-shifted analogue was known to Biane; in particular the second-named author witnessed a presentation of Biane in Spring 2008 in which this kind of result was stated by referring to computer simulations similar to the one from Fig. 4, see also [40] in the context of Gaussianity of fluctuations. Nevertheless, it seems that this non-shifted version was never explicitly stated in the existing literature and for this reason it was overlooked by the scientific community. For example, the authors of [33] cite the work of Biane but do not seem to be aware of a partial overlap of their result with [4].

\subsubsection{Example: random staircase tableaux}

The assumptions of the above Theorem 1.1 are fulfilled for the sequence

$$
\xi^{(k)}=\Delta_{k} \in \mathcal{S P}{ }_{n_{k}}
$$

of staircase strict partitions, cf. Eq. (5), with $n_{k}=\left(\begin{array}{c}k+1 \\ 2\end{array}\right)$ and the limit shape

$$
\Omega_{1}(x)= \begin{cases}2-|x| & \text { for }|x| \leq 1 \\ |x| & \text { for }|x|>1\end{cases}
$$

Theorem 1.1 is applicable and, as we shall see in Sect. 6.3.7, in this case the limit surface

$$
F(x, y)=L(x, y) \quad \text { for } 0 \leq y \leq x \leq 1
$$

is the restriction of the function $L$ described by Pittel and Romik [33, Sect. 1.1]. In this way we recover a part of the result of Linusson et al. [25, Theorem 3.8] who proved a stronger version of this result (the authors of [25] used convergence with respect 
to a stronger topology given by the pointwise convergence of the entries of tableaux towards the limit surface $F$ ). Note that the latter paper contains also large deviations results which do not seem to be accessible by out methods.

\subsection{The second example: asymptotics of shifted Schur-Weyl measures}

\subsubsection{Shifted RSK correspondence}

Let us fix some positive integers $n$ and $d$. We consider the ordered set

$$
\mathbb{A}_{d}:=\{1<1<2<2<\cdots<d<d\} .
$$

In the following we will use shifted RSK correspondence [13,36,49] in a very specific context (with the notations of [36, Theorem 8.1] this corresponds to the case when the circled matrix $\left(a_{i j}\right)_{i \in[n], j \in[d]}$ is such that $\sum_{j} a_{i j}=1$ for any $\left.i \in[n]\right)$. In this context shifted RSK is a bijection between:

- the set

$$
\Omega_{n, d}:=\left\{\mathbf{w}=\left(w_{1}, \ldots, w_{n}\right): w_{1}, \ldots, w_{n} \in \mathbb{A}_{d}\right\}
$$

of words of length $n$, and

- pairs $(P, Q)$ of (generalized) tableaux of the same shape $\xi \in \mathcal{S P}_{n}$ which fulfil the following conditions.

The insertion tableau $P$ is a generalized shifted tableau which means that it is a filling of the boxes of $\xi$ with the elements of $\mathbb{A}_{d}$ which is weakly increasing along the rows and along the columns; furthermore each circled symbol appears in each row at most once, and each non-circled symbol appears in each column at most once.

The recording tableau $Q$ is a filling of the boxes of $\xi$ with the elements of the set $[n]:=\{1, \ldots, n\}$ with the property that each element of $[n]$ appears exactly once; furthermore, each row and each column is increasing. Additionally, each non-diagonal entry of the tableau can be circled or not; each diagonal entries is non-circled.

We consider the discrete probability space $\Omega_{n, d}$ equipped with the uniform measure. We are interested in the probability distribution of the random variable $\xi=\xi(\mathbf{w})$. Since shifted RSK correspondence is a bijection, this probability distribution on $\mathcal{S} \mathcal{P}_{n}$-called shifted Schur-Weyl measure-is explicitly given by

$$
\begin{aligned}
& \mathbb{P}_{n, d}^{S W}(\xi) \\
& \quad=\frac{\#\left(\text { generalized shifted tableaux of shape } \xi \text { with entries in } \mathbb{A}_{d}\right) \cdot g^{\xi}}{2^{\ell(\xi)} d^{n}},
\end{aligned}
$$

where $\ell(\xi)$ denotes the number of non-zero parts of the partition $\xi$ and $g^{\xi}:=\left|\mathcal{T}_{\xi}\right|$ is the number of shifted standard tableaux of shape $\xi$. This probability distribution also 
has a natural representation-theoretic interpretation which we will be discussed later in Sect. 6.2.

\subsubsection{Asymptotics of shifted Schur-Weyl measures}

Usually we draw boxes which constitute a shifted Young diagram $\xi \in \mathcal{S} \mathcal{P}_{n}$ as unit squares. However, as we already mentioned, for asymptotic problems it might be beneficial to draw them as squares of side $\frac{1}{\sqrt{2 n}}$ so that the total area occupied by the boxes is equal to $\frac{1}{2}$.

The following result states that random strict partitions distributed according to shifted Schur-Weyl measures with carefully chosen parameters converge (after the rescaling of boxes described above) in probability towards some explicit limit shapes. The analogue of this result for non-shifted Schur-Weyl measures is due to Biane [3].

Theorem 1.3 (Law of large numbers for shifted Schur-Weyl measures)

Let $\left(d_{n}\right)$ be a sequence of positive integers with the property that the limit

$$
c:=\lim _{n \rightarrow \infty} \frac{\sqrt{n}}{d_{n}}
$$

exists.

Then there exists a function $\Omega_{c}^{S W}: \mathbb{R}_{+} \rightarrow \mathbb{R}_{+}$with the property that for each $\epsilon>0$

$$
\lim _{n \rightarrow \infty} \mathbb{P}_{n, d_{n}}^{S W}\left\{\xi \in \mathcal{S P} \mathcal{P}_{n}: \sup _{x \geq 0}\left|\omega_{\frac{1}{\sqrt{2 n}} \xi}(x)-\Omega_{c}^{S W}(x)\right|>\epsilon\right\}=0
$$

The proof is postponed to Sect. 6.2; the exact form of the limit curve will be discussed in Sect. 7.3.

This result is illustrated on Figs. 5 and 6.

\subsubsection{Asymptotics of the insertion tableaux $P$}

We continue the discussion of shifted RSK correspondence from Sect. 1.7.1. If we ignore that some of the entries of the insertion tableau $P=P(\mathbf{w})$ are circled, we may represent $P$ as a stack of cubes, just like we did it in Sect. 1.6.1. This time, however, we rescale all dimensions of the unit cubes by the factor $\frac{d}{n}$.

The following result states that such rescaled random surfaces converge in probability to some universal surface $\mathcal{P}$ in the sense of level curves. This result is illustrated by a computer simulation on Fig. 7 .

Corollary 1.4 (Law of Large Numbers for insertion tableaux) There exists a function

$$
\mathcal{P}:\{(X, Y): 0 \leq Y \leq X\} \rightarrow \mathbb{R}_{+}
$$




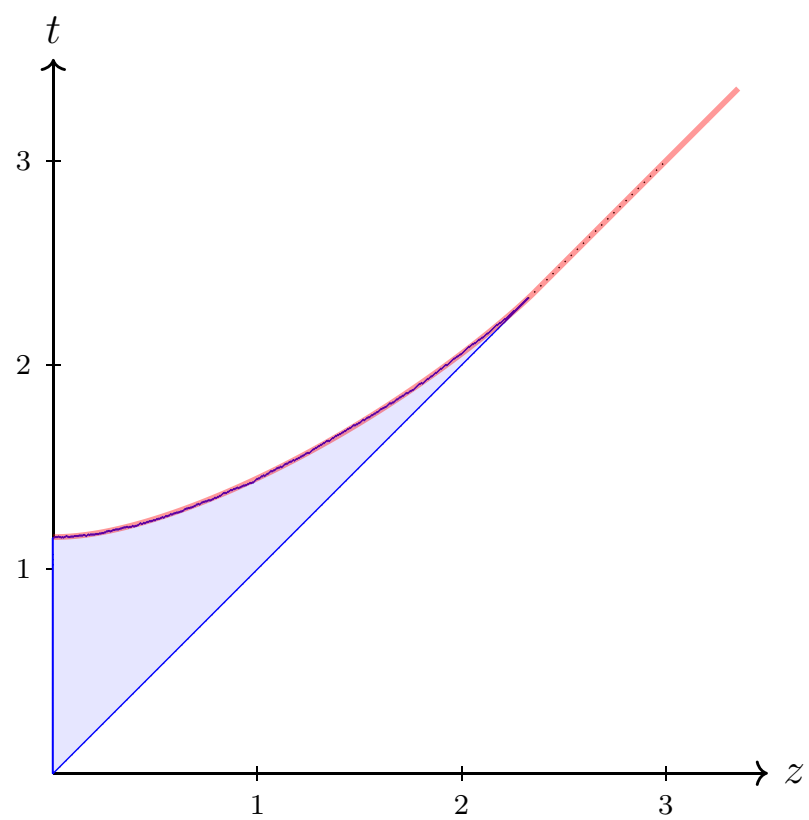

Fig. 5 The thick red line: the shape $\Omega_{c}^{\mathrm{SW}}$ for the special case $c=1$ obtained from (60) by numerical integration. The blue area: scaled down random shifted partition (shown in the shifted Russian convention) sampled for the shifted Schur-Weyl measure $\mathbb{P}_{n, d}^{\mathrm{SW}}$ for $n=80,000, d=283$ and $c=\frac{\sqrt{n}}{d} \approx 1$ (color figure online)

and the corresponding a family of level curves (drawn in the Russian convention) indexed by $\alpha>0$, defined for $z \geq 0$ by

$$
\Omega_{\alpha}^{\mathcal{P}}(z)=\sup \{x+y: 0 \leq y \leq x \text { and } x-y=z \text { and } \mathcal{P}(x, y) \leq \alpha\}
$$

with the following property.

For any sequence $\left(d_{n}\right)$ of positive integers such that

$$
\lim _{n \rightarrow \infty} \frac{d_{n}}{n}=0
$$

we have that

$$
\lim _{n \rightarrow \infty} \mathbb{P}\left\{w \in \Omega_{n, d}: \sup _{x \geq 0}\left|\omega_{\frac{d}{n}}(P(w))_{\leq \alpha \frac{n}{d}}(x)-\Omega_{\alpha}^{\mathcal{P}}(x)\right|>\epsilon\right\}=0
$$

(for legibility we write $d=d_{n}$ ) holds true for any $\epsilon>0$ and any level $\alpha$ such that

$$
0<\alpha<\liminf _{n \rightarrow \infty} \frac{d_{n}^{2}}{n} .
$$

The proof is postponed to Sect. 6.2. 


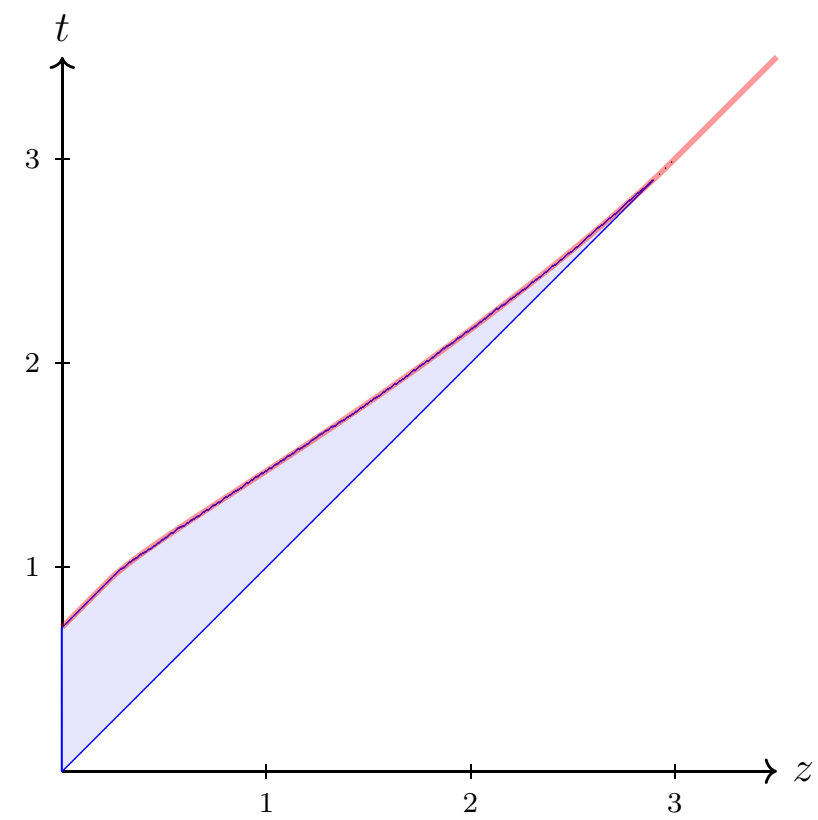

Fig. 6 The thick red line: the shape $\Omega_{c}^{\mathrm{SW}}$ for the special case $c=2$ obtained from (60) by numerical integration. The blue area: scaled down random shifted partition (shown in the shifted Russian convention) sampled for the shifted Schur-Weyl measure $\mathbb{P}_{n, d}^{S W}$ for $n=80,000, d=141$ and $c=\frac{\sqrt{n}}{d} \approx 2$ (color figure online)

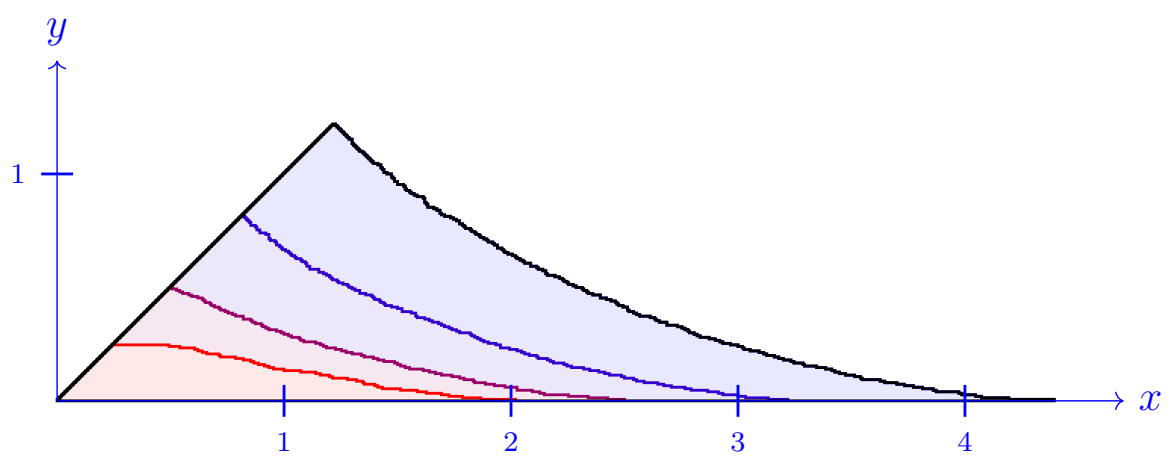

Fig. 7 Insertion tableau obtained by applying shifted version of RSK algorithm to a random word of length $n=45,000$ in the alphabet $\mathbb{A}_{d}$ with $d=300$. The boxes were drawn as squares of side $\frac{d}{n}=\frac{1}{150}$. In the context of Corollary 1.4 the Young diagram corresponds to the boxes with the rescaled height at most $t=\frac{d^{2}}{n}=2$. The level curves indicate positions of the boxes with rescaled height at most $t$ with: $\bullet$ the blue curve: $t=1, \bullet$ the burgundy curve: $t=\frac{1}{2}, \bullet$ the red curve: $t=\frac{1}{4}$ (color figure online)

\subsubsection{Asymptotics of recording tableaux $Q$}

Again, if we ignore that some of the entries of the recording tableau $Q=Q(\mathbf{w})$ are circled, we may represent $Q$ as a stack of cubes, just like we did it in Sect. 1.6.1. This 


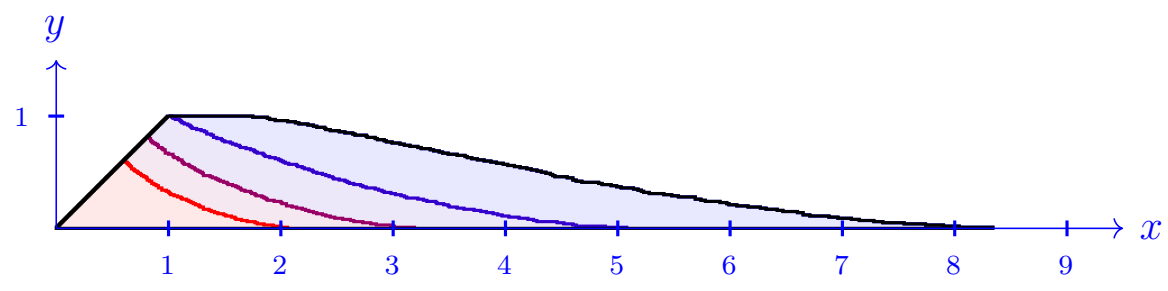

Fig. 8 Recording tableau obtained by applying shifted version of RSK algorithm to a random word of length $n=40,000$ in the alphabet $\mathbb{A}_{d}$ with $d=100$. The boxes were drawn as squares of side $\frac{1}{d}=\frac{1}{100}$. In the context of Corollary 1.5 the Young diagram corresponds to the boxes with the rescaled height at most $t=\frac{n}{d^{2}}=2$. The level curves indicate positions of the boxes with rescaled height at most $t$ with: $\bullet$ blue curve: $t=1$, burgundy curve: $t=\frac{1}{2} \bullet$ red curve: $t=\frac{1}{4}$ (color figure online)

time, we rescale the unit boxes on the plane by the factor $\frac{1}{d}$, and rescale the height of the cubes by the factor $\frac{1}{d^{2}}$.

The following result states that such rescaled random surfaces converge in probability to some universal surface $\mathcal{Q}$ in the sense of the level curves. This result is illustrated by a computer simulation on Fig. 8 .

Corollary 1.5 (Law of Large Numbers for recording tableaux) There exists a function

$$
\mathcal{Q}:\{(X, Y): 0 \leq Y \leq 1, Y \leq X\} \rightarrow \mathbb{R}_{+}
$$

and the corresponding a family of level curves (drawn in the Russian convention) indexed by $\alpha>0$, defined for $z \geq 0$ by

$$
\Omega_{\alpha}^{\mathcal{Q}}(z)=\sup \{x+y: 0 \leq y \leq x \text { and } y \leq 1 \text { and } x-y=z \text { and } \mathcal{Q}(x, y) \leq \alpha\}
$$

with the following property.

For any sequence $\left(d_{n}\right)$ of positive integers such that

$$
\lim _{n \rightarrow \infty} d_{n}=\infty
$$

we have that

$$
\lim _{n \rightarrow \infty} \mathbb{P}\left\{\boldsymbol{w} \in \Omega_{n, d}: \sup _{x \geq 0}\left|\omega_{\frac{1}{d}(Q(w))_{\leq \alpha d^{2}}}(x)-\Omega_{\alpha}^{\mathcal{Q}}(x)\right|>\epsilon\right\}=0
$$

(for legibility we write $d=d_{n}$ ) holds true for any $\epsilon>0$ and any level $\alpha$ such that

$$
0<\alpha<\liminf _{n \rightarrow \infty} \frac{n}{d_{n}^{2}}
$$

The proof is postponed to Sect. 6.2. 
Remark 1.6 Corollaries 1.4 and 1.5 have non-shifted analogues which concern the asymptotic shapes of the insertion and the recording tableaux when the usual (nonshifted) RSK correspondence is applied to a random sequence of length $n$ with the entries selected from the finite set $[d]$. These analogues follow from the results of Biane [3, Theorem 3] in a way similar to the one presented in the proofs of Corollaries 1.4 and 1.5. Somewhat surprisingly, it seems that these non-shifted analogues were not stated explicitly in the existing literature.

The results of the current paper can be also used to show that the fluctuations of the random surfaces corresponding to the insertion and the recording tableaux around the limit shapes are Gaussian.

\section{Spin representations and random strict partitions}

We are ready now to present the abstract general theory which provides the link between asymptotics of spin characters and the corresponding random strict partitions.

\subsection{Character ratios}

For Reader's convenience we collected some very basic facts and notations from the spin representation theory of the symmetric groups in "Appendix A". Nevertheless, most of these facts will not be used in the following. The bare minimal necessary knowledge is condensed in "Appendix A.7".

If $\psi: \mathbb{C S}_{n}^{-} \rightarrow \mathrm{GL}(V)$ is a spin representation and $\pi \in \mathcal{O P} \mathcal{P}_{n}$ is an odd partition, we define the corresponding character ratio

$$
\chi^{\psi}(\pi):=\operatorname{tr}_{V} \psi\left(c^{\pi}\right)=\frac{\operatorname{Tr} \psi\left(c^{\pi}\right)}{\operatorname{dim} V}
$$

as the (normalized) character of $\psi$ evaluated on any element $c^{\pi} \in C_{\pi}^{+}$which belongs to the conjugacy class which corresponds to $\pi$, cf. "Appendix A.7". Above

$$
\operatorname{tr}_{V}=\frac{1}{\operatorname{dim} V} \operatorname{Tr}
$$

denotes the normalized trace.

For $\xi \in \mathcal{S P}_{n}$ and $\pi \in \mathcal{O} \mathcal{P}_{n}$ we denote by

$$
\chi^{\xi}(\pi)=\frac{\phi^{\xi}(\pi)}{\phi^{\xi}\left(1^{n}\right)}
$$

the character ratio (8) which corresponds to (any) irreducible spin representation given by $\xi$, cf. "Appendix A.7". 


\subsection{Random strict partitions and reducible representations}

Let $\psi: G \rightarrow \operatorname{GL}(V)$ be a representation of a finite group $G$ and let

$$
V=\bigoplus_{\xi \in \widehat{G}} n_{\xi} V^{\xi}
$$

be its decomposition into irreducible components. Above, $n_{\xi} \in\{0,1,2, \ldots\}$ denotes the multiplicity of the irreducible component $V^{\xi}$. We define a probability measure on the set $\widehat{G}$ of the irreducible representations of $G$ given by

$$
\mathbb{P}^{V}(\xi):=\frac{n_{\xi} \operatorname{dim} V^{\xi}}{\operatorname{dim} V}
$$

which can be interpreted as the probability distribution of a random irreducible component of $V$.

The main results of the current paper concern the random shifted Young diagrams given by the above construction in the special case when $G=\widetilde{\mathfrak{S}}_{n}$ is the spin group and $V$ is its reducible spin representation. Equivalently, in order to ensure we deal with a spin representation, we may consider the analogue of the above construction in which instead of a group representation of $\widetilde{\mathfrak{S}}_{n}$ we use an algebra representation of $\mathbb{C S}_{n}^{-}$.

Due to the correspondence between the irreducible representations of $\mathbb{C S}_{n}^{-}$and strict partitions of $n$, the measure $\mathbb{P}^{V}$ can be equivalently viewed as a probability measure on $\mathcal{S P}_{n}$, see Sect. 2.3 for some technical details.

Example 2.1 (Strict Plancherel measure) The vector space $V:=\mathbb{C S}_{n}^{-}$admits a natural action of the spin group algebra $\mathbb{C S}_{n}^{-}$by left multiplication which can be regarded as an analogue of the left-regular representation of a group.

The corresponding probability measure, called strict (or shifted) Plancherel measure $[5,16,18]$, is given by

$$
\mathbb{P}_{n}^{\text {Plancherel }}(\xi)=\mathbb{P}^{\mathbb{C S}_{n}^{-}}(\xi)=\frac{2^{n-\ell(\xi)}\left(g^{\xi}\right)^{2}}{n !} \quad \text { for } \xi \in \mathcal{S P}_{n}
$$

where $g^{\xi}=\left|\mathcal{T}_{\xi}\right|$ denotes the number of shifted standard tableaux with shape given by the shifted Young diagram $\xi$.

Equivalently, (11) is the probability distribution of the common shape of the two shifted tableaux associated via shifted Robinson-Schensted correspondence $[36,49]$ to a uniformly random circled permutation in $n$ letters. 


\subsection{Random strict partitions, revisited}

Formally speaking, the construction from Sect. 2.2 associates to a given (reducible) representation $V$ of $\mathbb{C S}_{n}^{-}$a probability measure $\mathbb{P}^{V}$ on the set of irreducible representations (or irreducible characters) of $\mathbb{C S}_{n}^{-}$which can be identified with the set

$$
\begin{aligned}
& \mathcal{S P}_{n}^{+} \sqcup \mathcal{S P}_{n}^{-} \sqcup \mathcal{S P}_{n}^{-} \\
& \quad=\left\{\phi^{\xi}: \xi \in \mathcal{S P}_{n}^{+}\right\} \cup\left\{\phi_{+}^{\xi}: \xi \in \mathcal{S P}_{n}^{-}\right\} \cup\left\{\phi_{-}^{\xi}: \xi \in \mathcal{S P}_{n}^{-}\right\}
\end{aligned}
$$

in which each element of $\mathcal{S \mathcal { P } _ { n } ^ { - }}$ is counted twice, thus it is not equal to $\mathcal{S \mathcal { P } _ { n }}$. However, if we identify the two copies of $\mathcal{S P}_{n}^{-}$by identifying the characters $\phi_{ \pm}^{\xi}$ for $\xi \in \mathcal{S P}^{-}$ then $\mathbb{P}^{V}$ becomes, as required, a probability measure on $\mathcal{S P}_{n}$ given by

$$
\mathbb{P}^{V}(\xi)= \begin{cases}\mathbb{P}^{V}\left(\phi^{\xi}\right) & \text { if } \xi \in \mathcal{S P}^{+} \\ \mathbb{P}^{V}\left(\phi_{+}^{\xi}\right)+\mathbb{P}^{V}\left(\phi_{-}^{\xi}\right) & \text { if } \xi \in \mathcal{S P}^{-}\end{cases}
$$

An alternative solution to the above minor difficulty is to start with a reducible superrepresentation of $\mathbb{C S}_{n}^{-}$and then to decompose it into irreducible superrepresentations which directly gives rise to a probability distribution on strict partitions.

\subsection{Random strict partitions, alternative viewpoint}

If $V$ is a representation of of $\mathbb{C S}_{n}^{-}$then (keeping in mind (13)) the following equality between functions on $\mathcal{O P}$, holds true:

$$
\chi^{V}=\sum_{\xi \in \mathcal{S} \mathcal{P}_{n}} \mathbb{P}^{V}(\xi) \chi^{\xi}
$$

Thanks to Lemma 2.2 below it follows that the coefficients $\left(\mathbb{P}^{V}(\xi)\right)$ of this expansion are uniquely determined by (14).

Lemma 2.2 The family of character ratios

$$
\left\{\chi^{\xi}: \xi \in \mathcal{S} \mathcal{P}_{n}\right\}
$$

forms a linear basis of the space of (complex-valued) functions on $\mathcal{O P}_{n}$.

Proof The right-hand side of (12) is the complete collection of the characters of $\mathbb{C S}_{n}^{-}$ hence it forms a linear basis of the space of complex-valued functions on the set of conjugacy classes $C_{\pi}^{+}$over $\pi \in \mathcal{O P}_{n} \cup \mathcal{S P}_{n}^{-}$.

It follows that

$$
\left\{\phi^{\xi}: \xi \in \mathcal{S P}_{n}\right\}
$$


spans the space of complex-valued functions on the set of conjugacy classes $C_{\pi}^{+}$over $\pi \in \mathcal{O} \mathcal{P}_{n}$. By the identity $\left|\mathcal{S P}_{n}\right|=\left|\mathcal{O} \mathcal{P}_{n}\right|$, its cardinality matches the dimension hence (15) is a linear basis of the latter space.

Since

$$
\chi^{\xi}=\frac{1}{\phi^{\xi}\left(1^{n}\right)} \phi^{\xi},
$$

the claim follows immediately.

Equality (14) can be viewed as an alternative definition of the probabilities $\mathbb{P}^{V}(\xi)$ as coefficients of the expansion of $\chi^{V}$ in the linear basis $\left(\chi^{\xi}\right)$. This viewpoint has interesting consequences.

Firstly, in order to state the results of the current paper we do not need the representation $V$ and it is enough to speak about the corresponding character ratio $\chi^{V}$. The property of approximate factorization (Definition 2.3) is, in fact, not a property of a sequence of representations but of the corresponding sequence of character ratios.

Secondly, for a superrepresentation of $\mathbb{C S}_{n}^{-}$, the construction from Sect. 2.2 gives a probability measure directly on $\mathcal{S P}_{n}$, without the difficulties discussed in Sect. 2.3. Passage from the framework of representations to the framework of superrepresentations implies that we should replace the family of characters (12) by the characters of the irreducible superrepresentations

$$
\left\{\phi^{\xi}: \xi \in \mathcal{S P}_{n}^{+}\right\} \cup\left\{\phi_{+}^{\xi}+\phi_{-}^{\xi}: \xi \in \mathcal{S P}_{n}^{-}\right\}
$$

This change does not create any difficulties because the corresponding family of character ratios (viewed as functions on $\mathcal{O P}_{n}$ ) remains the same. By revisiting Eq. (14) in the new context of superrepresentations we see that it still remains valid; this shows that that the probability measure on $\mathcal{S P}_{n}$ associated to a (super)representation $V$ remains the same, no matter if we regard $V$ as a superrepresentation or as a representation.

Thirdly, the (super)representation theory of the spin symmetric groups $\mathfrak{S}_{n}$ is known to be Morita equivalent to the (super)representation theory of Hecke-Clifford algebra $\mathcal{H}_{n}=\mathcal{C} l_{n} \rtimes \mathbb{C S}_{n}$. In the context of the (super)representations of $\mathcal{H}_{n}$ it still makes sense to speak about the character ratios $\chi$ as functions on $\mathcal{O P} \mathcal{P}_{n}$; in this way the results of the current paper can be reformulated in the language of the (super)representations of $\mathcal{H}_{n}$ and the corresponding probability measures.

\subsection{Hypothesis of the main results: approximate factorization of characters}

In this section we will present the hypothesis of the main results of the current paper, Theorems 2.5 and 2.6.

\subsubsection{Cumulants of characters}

We define a product of two odd partitions as their concatenation, followed by arranging the entries in a weakly decreasing manner. In this way the set $\mathcal{O P}$ of odd partitions 
becomes a commutative monoid with the unit given by the empty partition $\emptyset$. We consider the algebra of odd partitions $\mathbb{C}[\mathcal{O P}]$ which-as a vector space-is defined as the set of formal linear combinations of odd partitions; the product corresponds to the above monoid structure via distributivity of multiplication. Any function $\chi: \mathcal{O P} \rightarrow \mathbb{C}$ on odd partitions extends by linearity to a linear map $\chi: \mathbb{C}[\mathcal{O P}] \rightarrow \mathbb{C}$ on the odd partition algebra.

A convenient way to encode the information about a function $\chi: \mathcal{O P} \rightarrow \mathbb{C}$ with the property that $\chi(\emptyset)=1$ is to use cumulants. More specifically, for partitions $\pi^{1}, \ldots, \pi^{\ell} \in \mathcal{O P}$ we define their cumulant (with respect to $\chi$ )

$$
\begin{aligned}
& \kappa_{\ell}^{\chi}\left(\pi^{1}, \ldots, \pi^{\ell}\right):=\left.\frac{\partial^{\ell}}{\partial t_{1} \cdots \partial t_{\ell}} \log \chi\left(e^{t_{1} \pi^{1}+\cdots+t_{\ell} \pi^{\ell}}\right)\right|_{t_{1}=\cdots=t_{\ell}=0} \\
& \quad=\left[t_{1} \cdots t_{\ell}\right] \log \chi\left(e^{t_{1} \pi^{1}+\cdots+t_{\ell} \pi^{\ell}}\right)
\end{aligned}
$$

as a coefficient in the Taylor series of an analogue of the logarithm of the (multidimensional) Laplace transform. The operations on the right-hand side should be understood in the sense of formal power series with values in the odd partitions algebra $\mathbb{C}[\mathcal{O P}]$. For example,

$$
\begin{aligned}
\kappa_{1}\left(\pi^{1}\right) & =\chi\left(\pi^{1}\right), \\
\kappa_{2}\left(\pi^{1}, \pi^{2}\right) & =\chi\left(\pi^{1} \pi^{2}\right)-\chi\left(\pi^{1}\right) \chi\left(\pi^{2}\right) .
\end{aligned}
$$

Informally speaking, the cumulants $\kappa_{\ell}^{\chi}$ (for $\ell \geq 2$ ) quantify the extent to which $\chi: \mathcal{O P} \rightarrow \mathbb{C}$ fails to be a semigroup homomorphism (with respect to the multiplication).

We denote by $\mathcal{O P} \mathcal{P}_{\leq n}:=\bigcup_{0 \leq m \leq n} \mathcal{O \mathcal { P } _ { m }}$ the set of odd partitions of size smaller or equal than $n$. We will apply the above construction of cumulants to the special case when $V$ is a (reducible) spin representation of $\widetilde{\mathfrak{S}}_{n}$ and $\chi=\chi^{V}$ is the character ratio defined on $\mathcal{O P} \mathcal{P}_{\leq n}$ by extending the domain of (8) by padding the partition $\pi$ with additional ones:

$$
\chi^{V}(\pi):=\operatorname{tr}_{V} \psi\left(\pi, 1^{n-|\pi|}\right)=\frac{\operatorname{Tr} \psi\left(\pi, 1^{n-|\pi|}\right)}{\operatorname{dim} V} \quad \text { for } \pi \in \mathcal{O P} \mathcal{P}_{\leq n}
$$

Note that so defined $\chi^{V}$ is well-defined only on the set $\mathcal{O P} \mathcal{P}_{\leq n}$; in this way the cumulant (16) is well-defined as long as $\left|\pi^{1}\right|+\cdots+\left|\pi^{\ell}\right| \leq n$.

\subsubsection{Approximate factorization of characters}

For a partition $\pi=\left(\pi_{1}, \ldots, \pi_{\ell}\right)$ with $\pi_{1}, \ldots, \pi_{\ell} \geq 1$ we define its length

$$
\|\pi\|:=|\pi|-\ell=\left(\pi_{1}-1\right)+\cdots+\left(\pi_{\ell}-1\right)
$$

as the difference of its size and its number of parts. 
Definition 2.3 Assume that for each integer $n \geq 1$ we are given a spin representation $\psi^{(n)}: \widetilde{\mathfrak{S}}_{n} \rightarrow \operatorname{GL}\left(V^{(n)}\right)$. We say that the sequence $\left(V^{(n)}\right)$ has approximate factorization property if for each $l \geq 1$ and all $\pi^{1}, \ldots, \pi^{\ell} \in \mathcal{O P}$ such that each $\pi^{i}=\left(2 k_{i}+1\right)$ is an odd partition which consists of exactly one part, we have that

$$
\kappa_{\ell}^{V^{(n)}}\left(\pi^{1}, \ldots, \pi^{\ell}\right)=O\left(n^{-\frac{\left\|\pi^{1}\right\|+\cdots+\left\|\pi^{\ell}\right\|+2(\ell-1)}{2}}\right) \quad \text { for } n \rightarrow \infty .
$$

Example 2.4 We continue Example 2.1. The vector space $\mathbb{C S}_{n}^{-}$is the image of the left-regular representation $\mathbb{C}_{\mathfrak{S}}$ under the projection $\frac{1-z}{2}$. Since the character of the left-regular representation vanishes on all group elements (except for the identity 1), it follows that the character of $\mathbb{C S}_{n}^{-}$vanishes on all group elements, except for 1 and $z$, and hence the corresponding character ratio is given by

$$
\chi^{\mathbb{C S}_{n}^{-}(\pi)}= \begin{cases}1 & \text { if } \pi=\left(1^{n}\right) \\ 0 & \text { otherwise }\end{cases}
$$

for any $\pi \in \mathcal{O} \mathcal{P}_{n}$. We extend the domain of the character ratio by (17) and obtain

$$
\chi^{\mathbb{C S}_{n}^{-}}(\pi)= \begin{cases}1 & \text { if } \pi=\left(1^{k}\right) \text { for some } 0 \leq k \leq n, \\ 0 & \text { otherwise }\end{cases}
$$

for any $\pi \in \mathcal{O P} \mathcal{P}_{\leq n}$.

Since $\kappa_{1}^{\mathbb{C S}_{n}^{-}}(\pi)=\chi^{\mathbb{C S}_{n}^{-}}(\pi)$, we just calculated the first cumulant as well.

Since the map (19) is a homomorphism (in the somewhat restricted sense that $\chi^{\mathbb{C S}_{n}^{-}}\left(\pi^{1} \pi^{2}\right)=\chi^{\mathbb{C S}_{n}^{-}}\left(\pi^{1}\right) \chi^{\mathbb{C S}_{n}^{-}}\left(\pi^{2}\right)$ for all $\pi^{1}, \pi^{2} \in \mathcal{O P}$ such that $\left|\pi^{1}\right|+\left|\pi^{2}\right| \leq$ $n$ ), it follows immediately that all higher cumulants $\kappa_{\ell}^{\mathbb{C S}_{n}^{-}}$(for $\ell \geq 2$ ) vanish.

Now it is easy to check that the sequence of representations $\left(\mathbb{C S}_{n}^{-}\right)$indeed has approximate factorization property.

We will construct a whole class of examples later in Sects. 6.2 and 6.3.

\subsection{Free cumulants}

It was noticed by Biane [3,4] that for asymptotic problems it is convenient to parametrize the set of Young diagrams by free cumulants, quantities which originate in the random matrix theory and Voiculescu's free probability [30]. We review these quantities in the following.

For a continual Young diagram $\omega$ we consider a function $\sigma_{\omega}: \mathbb{R} \rightarrow \mathbb{R}_{+}$given by

$$
\sigma_{\omega}(z):=\frac{\omega(z)-|z|}{2}
$$

which can be viewed as the density of a measure on $\mathbb{R}$. 
If $\omega=\omega_{r \lambda}$ is the profile of a rescaled Young diagram for a Young diagram $\lambda$ and $r>0$ then the total weight of this measure

$$
\int_{\mathbb{R}} \sigma_{r \lambda}(z) \mathrm{d} z=r^{2}|\lambda|
$$

is equal to the area of the rescaled Young diagram $r \lambda$ (there are $|\lambda|$ boxes, each is a square of side $r$ ).

Similarly, if $\omega=\omega_{r \xi}: \mathbb{R} \rightarrow \mathbb{R}_{+}$is the profile of a rescaled shifted Young diagram $\xi$ then

$$
\int_{\mathbb{R}} \sigma_{r \xi}(z) \mathrm{d} z=2 r^{2}|\xi|
$$

is the double of the area of the rescaled shifted Young diagram $r \xi$ (the additional factor 2 appears because we extended the domain of the profile to the whole real line).

For a given continual Young diagram $\omega$ and an integer $n \geq 2$ we define the rescaled moment of the measure $\sigma_{\omega}$ :

$$
S_{n}=S_{n}(\omega)=(n-1) \int_{\mathbb{R}} z^{n-2} \sigma_{\omega}(z) \mathrm{d} z
$$

Then the sequence of free cumulants $R_{2}, R_{3}, \ldots$ is defined by

$$
R_{n}=R_{n}(\omega)=\sum_{l \geq 1} \frac{1}{l !}(-n+1)^{l-1} \sum_{\substack{k_{1}, \ldots, k_{l} \geq 2, k_{1}+\cdots+k_{l}=n}} S_{k_{1}} \cdots S_{k_{l}} .
$$

Conversely, the sequence of free cumulants determines uniquely the corresponding sequence of moments $S_{2}, S_{3}, \ldots$ by the identity

$$
S_{n}=\sum_{l \geq 1} \frac{1}{l !}(n-1)^{\downarrow l-1} \sum_{\substack{k_{1}, \ldots, k_{l} \geq 2, k_{1}+\cdots+k_{l}=n}} R_{k_{1}} \cdots R_{k_{l}},
$$

where $m^{\downarrow k}=m(m-1) \cdots(m-k+1)$ denotes the falling power, see [7, Sect. 3.2 and Proposition 2.2].

\subsection{The first main result: random strict partitions concentrate around some limit shape}

In the following, in order to keep the notation lightweight, we will write $\chi^{(n)}, \kappa_{\ell}^{(n)}$, $\mathbb{P}^{(n)}$ instead of $\chi^{V^{(n)}}, \kappa_{\ell}^{V^{(n)}}, \mathbb{P}^{V^{(n)}}$, etc.

Theorem 2.5 Assume that for each integer $n \geq 1$ we are given a spin representation $\psi^{(n)}: \widetilde{\mathfrak{S}}_{n} \rightarrow \operatorname{GL}\left(V^{(n)}\right)$ and assume that the sequence $\left(V^{(n)}\right)$ fulfils the approximate factorization property (Definition 2.3). 
Additionally, we require that for all odd numbers $k, l \geq 3$ the following limits exist:

$$
\begin{aligned}
\boldsymbol{r}_{k+1} & :=\lim _{n \rightarrow \infty} n^{\frac{k-1}{2}} \chi^{(n)}(k), \\
\boldsymbol{k}_{k+1, l+1}^{\bullet} & :=\lim _{n \rightarrow \infty} n^{\frac{k+l}{2}} \cdot 2 \cdot \kappa_{2}^{(n)}((k),(l)) \\
& =\lim _{n \rightarrow \infty} n^{\frac{k+l}{2}} \cdot 2 \cdot\left(\chi^{(n)}(k, l)-\chi^{(n)}(k) \chi^{(n)}(l)\right)
\end{aligned}
$$

(note that Definition 2.3 implies already that the expressions under the limits are $O(1)$ ); and that and that the sequence $\boldsymbol{r}_{2}, \boldsymbol{r}_{4}, \ldots$ grows at most like a geometric sequence:

$$
\limsup _{k \rightarrow \infty} \sqrt[k]{\left|\boldsymbol{r}_{k}\right|}<\infty
$$
$\mathbb{P}(n)$

We denote by $\xi^{(n)}$ the random shifted Young diagram with the distribution given by

Then the sequence of rescaled shifted Young diagrams $\frac{1}{\sqrt{n}} \xi^{(n)}$ converges in probability towards some limit $\Omega$. In other words: there exists a unique continual Young diagram $\Omega: \mathbb{R} \rightarrow[0, \infty)$ ("the limit shape") with the property that for each $\epsilon>0$

$$
\lim _{n \rightarrow \infty} \mathbb{P}^{(n)}\left(\xi^{(n)} \in \mathcal{S} \mathcal{P}_{n}:\left\|\omega_{\frac{1}{\sqrt{n}} \xi^{(n)}}-\Omega\right\|>\epsilon\right)=0,
$$

where $\|\cdot\|$ denotes the supremum norm.

This limit shape $\Omega: \mathbb{R} \rightarrow \mathbb{R}_{+}$is uniquely determined by its free cumulants

$$
R_{k}(\Omega)= \begin{cases}\boldsymbol{r}_{k} & \text { if } k \text { is even } \\ 0 & \text { if } k \text { is odd }\end{cases}
$$

The proof of this result is postponed to Sect. 5.3. This theorem is analogous to a result of Biane [3, Corollary 1] who considered linear representations of the symmetric groups and the corresponding random (non-shifted) Young diagrams. The assumptions of Theorem 2.5 can be weakened to match the assumptions of the analogous result of Biane; for simplicity we decided to have the same assumptions for Theorems 2.5 and 2.6.

In Sect. 6.1 we will prove that in the special case considered in Example 2.4 when $V^{(n)}=\mathbb{C S}_{n}^{-}$is the spin part of the left-regular representation and the corresponding probability measure $\mathbb{P}^{(n)}$ is the shifted Plancherel measure, the limit curve $\Omega$ coincides with the Logan-Shepp \& Vershik-Kerov curve [26,48] which describes the limit shape of (non-shifted) random Young diagram distributed to the (non-shifted) Plancherel measure. In this case the proof is due to De Stavola [10, Sect. 4.5]; this result was conjectured earlier by the authors of [2]. 


\subsection{The second main result: Gaussian fluctuations}

Theorem 2.6 We keep the notations and the assumptions from Theorem 2.5.

(1) (Gaussian fluctuations of characters) Then the joint distribution of (any finite collection of) the centred random variables

$$
n^{\frac{k}{2}}\left(\chi^{\xi^{(n)}}(k)-\mathbb{E} \chi^{\xi^{(n)}}(k)\right), \quad k \in\{3,5,7,9, \ldots\}
$$

converges in distribution to a Gaussian distribution, where

$$
\chi^{\xi^{(n)}}(k)=\chi^{\xi^{(n)}}((k))=\chi^{\xi^{(n)}}((k, \underbrace{1, \ldots, 1}_{n-k \text { times }}))
$$

denotes the evaluation of the character ration on the odd partition $(k)$ which consists of a single part.

(2) (Gaussian fluctuations of shapes) Then the joint distribution of (any finite collection of) the random variables

$$
\sqrt{n} \int_{0}^{\infty} x^{2 k}\left(\omega_{\frac{1}{\sqrt{n}} D\left(\xi^{(n)}\right)}(x)-\Omega(x)\right) d x, \quad k \in\{1,2, \ldots\}
$$

converges in distribution to a centered Gaussian distribution, where $\Omega$ is the function provided by Theorem 2.5 .

The proof is postponed to Sect. 5.4. The explicit form of the covariance can be calculated thanks to Theorem 5.1. This result is analogous to the central limit theorem for random (non-shifted) Young diagrams proved by the second-named author [41] which was an extension of Kerov's Central Limit Theorem $[15,20]$ to the non-Plancherel case. Note also that the special case of the above result for the shifted Plancherel measure was proved by Ivanov [16] already in 2004.

The following sections are a preparation for the proofs of Theorems 2.5 and 2.6.

\section{The approximate factorization category}

The notion of approximate factorization of characters was introduced by the secondnamed author as a tool for proving Gaussianity of fluctuations of random Young diagrams related to representation theory and special functions [11,41]. We use this occasion to present this notion in a more abstract and more transparent framework.

\subsection{Filtered algebras}

In the usual definition of a filtered algebra $\mathcal{A}=\bigcup_{i \in \mathbb{Z}_{\geq 0}} \mathcal{F}_{i}$ the family $\left(\mathcal{F}_{i}\right)$ is indexed by non-negative integers. For this reason we will refer to such a filtered algebra as $\mathbb{Z}_{\geq 0}$-filtered algebra. The following is a slight extension of this concept. Note that 
each such a $\mathbb{Z}_{\geq 0}$-filtered algebra becomes a $\mathbb{Z}$-filtered algebra by setting $\mathcal{F}_{i}:=\{0\}$ for all negative integers $i<0$.

Definition 3.1 By a $\mathbb{Z}$-filtered algebra we will understand an algebra $\mathcal{A}$ together with a family (indexed by integers) of linear subspaces $\left(\mathcal{F}_{i}\right)_{i \in \mathbb{Z}}$ which is increasing: $\cdots \subseteq$ $\mathcal{F}_{-1} \subseteq \mathcal{F}_{0} \subseteq \mathcal{F}_{1} \subseteq \cdots \subseteq \mathcal{A}$, such that $\mathcal{A}=\bigcup_{i} \mathcal{F}_{i}$ and such that $\mathcal{F}_{i} \cdot \mathcal{F}_{j} \subseteq \mathcal{F}_{i+j}$ holds true for all $i, j \in \mathbb{Z}$. We will always assume that $\mathcal{A}$ has a unit and $1 \in \mathcal{F}_{0}$.

For $x \in \mathcal{A}$ its degree $\operatorname{deg}_{\mathcal{A}} x$ is defined as the minimal value of $i \in \mathbb{Z}$ such that $x \in \mathcal{F}_{i}$.

Often we do not need to distinguish between $\mathbb{Z}$ - and $\mathbb{Z}_{\geq 0}$-filtered algebras; in this case we will speak simply about filtered algebras.

\subsection{Examples of filtered algebras}

The following two examples will play an important role later on.

\subsubsection{The algebra $\mathcal{X}$ of sequences with polynomial growth}

For $i \in \mathbb{Z}$ we define

$$
\mathcal{F}_{i}:=\left\{\left(f_{1}, f_{2}, \ldots\right): \sup _{n}\left|f_{n}\right| n^{-\frac{i}{2}}<\infty\right\}
$$

to be the linear space of (real valued) sequences with growth at most $O\left(n^{\frac{i}{2}}\right)$. Then $\mathcal{X}:=\bigcup_{i} \mathcal{F}_{i}$ is a unital $\mathbb{Z}$-filtered commutative algebra with the multiplication given by the pointwise product. The unit $1=(1,1, \ldots) \in \mathcal{F}_{0}$ corresponds to the constant sequence.

\subsubsection{The algebra of odd partitions}

We revisit Sect. 2.5.1 and we equip the algebra $\mathbb{C}[\mathcal{O P}]$ of odd partitions with a $\mathbb{Z}$ filtration by setting

$$
\mathcal{H}_{i}=\operatorname{span}\{\pi \in \mathcal{O P}:-\|\pi\| \leq i\}
$$

for any $i \in \mathbb{Z}$.

It should be stressed that this filtration has a peculiar property that the degree of any element is a non-positive integer.

\subsection{The approximate factorization property}

Definition 3.2 Suppose that $\mathcal{A}$ and $\mathcal{B}$ are unital commutative algebras and $F: \mathcal{A} \rightarrow \mathcal{B}$ is a linear unital map. For $a_{1}, \ldots, a_{l} \in \mathcal{A}$ we define their cumulant 


$$
\begin{aligned}
& \kappa_{\ell}^{F}\left(a_{1}, \ldots, a_{\ell}\right):=\left.\frac{\partial^{\ell}}{\partial t_{1} \cdots \partial t_{\ell}} \log F\left(e^{t_{1} a_{1}+\cdots+t_{\ell} a_{\ell}}\right)\right|_{t_{1}=\cdots=t_{l}=0} \\
& \quad=\left[t_{1} \cdots t_{\ell}\right] \log F\left(e^{t_{1} a_{1}+\cdots+t_{\ell} a_{\ell}}\right) \in \mathcal{B}
\end{aligned}
$$

where the operations on the right-hand side should be understood in the sense of formal power series in the variables $t_{1}, \ldots, t_{\ell}$, cf. (16).

So defined cumulant is a coefficient in the expansion of an multidimensional Laplace transform, with the role of the expected value $\mathbb{E}$ played by the linear map $F$. For example,

$$
\left\{\begin{aligned}
\kappa_{1}^{F}\left(a_{1}\right) & =F\left(a_{1}\right), \\
\kappa_{2}^{F}\left(a_{1}, a_{2}\right) & =F\left(a_{1} a_{2}\right)-F\left(a_{1}\right) F\left(a_{2}\right), \\
\vdots &
\end{aligned}\right.
$$

correspond to the mean value and the covariance.

Definition 3.3 We say that a linear unital map $F: \mathcal{A} \rightarrow \mathcal{B}$ between filtered commutative algebras has the approximate factorization property if for all $\ell \geq 1$ and $a_{1}, \ldots, a_{\ell} \in \mathcal{A}$

$$
\operatorname{deg}_{\mathcal{B}} \kappa_{\ell}^{F}\left(a_{1}, \ldots, a_{\ell}\right) \leq\left(\operatorname{deg}_{\mathcal{A}} a_{1}\right)+\cdots+\left(\operatorname{deg}_{\mathcal{A}} a_{\ell}\right)-2(\ell-1)
$$

Remark 3.4 More generally, for an arbitrary $\alpha>0$ one can consider the notion of $\alpha$-approximate factorization property in which (28) is replaced by the condition

$$
\operatorname{deg}_{\mathcal{B}} \kappa_{\ell}^{F}\left(a_{1}, \ldots, a_{\ell}\right) \leq\left(\operatorname{deg}_{\mathcal{A}} a_{1}\right)+\cdots+\left(\operatorname{deg}_{\mathcal{A}} a_{\ell}\right)-\alpha(\ell-1)
$$

Clearly, Definition 3.3 is a special case corresponding to $\alpha=2$.

Some of the abstract general results which we present in the following remain true also for this more general notion (in particular, all results from Sects. 3.4 and 3.5). On the other hand, the results which concern specific examples (such as Observation 3.9 and Proposition 3.10) depend on the specific choice of $\alpha=2$.

In order to keep the notation lightweight we will not pursue further this more general setup.

\subsection{The approximate factorization category}

Lemma 3.5 If $\mathcal{A}, \mathcal{B}, \mathcal{C}$ are filtered unital commutative algebras and $F: \mathcal{A} \rightarrow \mathcal{B}$ and $G: \mathcal{B} \rightarrow \mathcal{C}$ have the approximate factorization property then their composition $G \circ$ $F: \mathcal{A} \rightarrow \mathcal{C}$ also has approximate factorization property.

This lemma appears in a rather concealed form in [41, Sect. 4.7, proof of the implication $(13) \Longrightarrow(14)$ ]; the main idea of the proof is to use the formula of Brillinger [6] in 
order to express the cumulants for the composition $G \circ F$ in terms of the cumulants for $G$ and cumulants for $F$.

Lemma 3.5 allows us to speak about the approximate factorization category which has filtered unital commutative algebras as objects and unital maps with the approximate factorization property as morphisms.

Lemma 3.6 Suppose that $F: \mathcal{A} \rightarrow \mathcal{B}$ has the approximate factorization property and additionally it preserves the degree, i.e. $\operatorname{deg}_{\mathcal{B}} F(a)=\operatorname{deg}_{\mathcal{A}}$ a for each $a \in \mathcal{A}$, and that $F$ is invertible as a linear map.

Then $F^{-1}: \mathcal{B} \rightarrow \mathcal{A}$ also has the approximate factorization property.

This lemma appears in a concealed form in [41, Sect. 4.7, proof of the implication (14) $\Longrightarrow(13)]$.

\subsection{Generators and approximate factorization}

Since we consider the setup of filtered algebras, the usual notion of generators of an algebra has to be adjusted accordingly.

Definition 3.7 Let $\mathcal{A}$ be a filtered algebra and let $X \subseteq \mathcal{A}$ be its subset. We say that $X$ generates $\mathcal{A}$ as a filtered algebra if each $a \in \mathcal{A}$ is a linear combination (with complex coefficients) of finite products of the form $x_{1} \cdots x_{\ell}$ for some $\ell \geq 0$ and $x_{1}, \ldots, x_{\ell} \in X$ such that

$$
\operatorname{deg} x_{1}+\cdots+\operatorname{deg} x_{\ell} \leq \operatorname{deg} a .
$$

The following simple result was proved by the second-named author [41, Corollary 19] in the specific setup of the Kerov-Olshanski algebra (with two distinct multiplicative structures). The proof did not use any specific properties of these filtered algebras and thus it remains valid also in this general context. Note that the original paper mistakenly does not mention the necessary condition (29); see also [11, Lemma 3.4] and the proceeding discussion.

Lemma 3.8 Let $F: \mathcal{A} \rightarrow \mathcal{B}$ be a linear unital map between filtered commutative algebras and let $X$ be a set which generates $\mathcal{A}$ as a filtered algebra.

If condition (28) holds true for all $\ell \geq 1$ and $a_{1}, \ldots, a_{\ell} \in X$ then it holds for arbitary $a_{1}, \ldots, a_{\ell} \in \mathcal{A}$; in other words $F$ has the approximate factorization property.

\subsection{Example: approximate factorization of characters revisited}

In Definition 2.3 we defined the approximate factorization property for a sequence of representations while above we used the same name in Definition 3.3 in the context of maps between filtered algebras. As we explain below, this is not a coincidence.

With the notations of Definition 2.3, let $\left(V^{(n)}\right)$ be a fixed sequence of representations and let $\left(\chi^{(n)}\right)$ be the corresponding sequence of the character ratios with $\chi^{(n)}: \mathcal{O P}_{\leq n} \rightarrow \mathbb{R}$. We extend the domain of $\chi^{(n)}$ in an arbitrary way so that 
$\chi^{(n)}: \mathcal{O P} \rightarrow \mathbb{R}$; for example we may set $\chi^{(n)}(\pi)=0$ if $|\pi|>n$. The information about this sequence of character ratios can be encoded by a single map

$$
\chi: \mathbb{C}[\mathcal{O P}] \rightarrow \mathcal{X}
$$

which is defined by

$$
\chi(\pi):=\left(\chi^{(1)}(\pi), \chi^{(2)}(\pi), \ldots\right) \quad \text { for } \pi \in \mathcal{O P} .
$$

Observation 3.9 The map (30) has the approximate factorization property (in the sense of Definition 3.3) if and only if the sequence of representations $\left(V^{(n)}\right)$ has the approximate factorization property (in the sense of Definition 2.3).

Proof We denote by $X \subset \mathcal{O P}$ the set of odd partitions which consist of exactly one part. This set generates $\mathcal{O P}$ as a commutative monoid. We shall identify any odd partition with the corresponding element of the odd partition algebra $\mathbb{C}[\mathcal{O P}]$; it is easy to check that $X \subset \mathbb{C}[\mathcal{O P}]$ generates the odd partition algebra $\mathbb{C}[\mathcal{O P}]$ as a filtered algebra.

Assume that $\left(V^{(n)}\right)$ has the approximate factorization property. The condition (18) implies that the assumptions of Lemma 3.8 are fulfilled for the map (30) and the aforementioned generating set $X$. It follows that (30) indeed has the approximate factorization property, as required.

The opposite implication is immediate.

\subsection{The motivating example: Gaussian fluctuations}

The following example will be our key tool for proving Gaussianity of various random variables.

For each $n \geq 1$ let $\left(\Omega_{n}, \mathfrak{F}_{n}, \mathbb{P}_{n}\right)$ be a probability space. Assume that $\mathcal{A}$ is a $\mathbb{Z}$-filtered commutative algebra such that each element $\mathbf{X} \in \mathcal{A}$ is a sequence $\mathbf{X}=\left(X_{1}, X_{2}, \ldots\right)$ where $X_{n}: \Omega_{n} \rightarrow \mathbb{R}$ is a random variable on the appropriate probability space with the property that its expected value $\mathbb{E}_{n} X_{n}$ is well-defined.

We assume that the unital map $\mathbb{E}: \mathcal{A} \rightarrow \mathcal{X}$ given by the expected value:

$$
\mathbb{E}:\left(X_{1}, X_{2}, \ldots\right) \mapsto\left(\mathbb{E}_{1} X_{1}, \mathbb{E}_{2} X_{2}, \ldots\right)
$$

is well-defined, i.e. it indeed takes values in $\mathcal{X}$.

Proposition 3.10 With the above notations, let us assume that $\mathbb{E}$ has the approximate factorization property. Let $\left\{\boldsymbol{X}_{1}, \ldots, \boldsymbol{X}_{l}\right\} \subset \mathcal{A}$ be a finite set; we denote $\boldsymbol{X}_{i}=\left(X_{i, 1}, X_{i, 2}, \ldots\right)$ and define a centered random variable

$$
y_{i, n}:=n^{\frac{1-\operatorname{deg} X_{i}}{2}} \cdot\left(X_{i, n}-\mathbb{E}_{n} X_{i, n}\right) .
$$

Assume that the limit of the covariance

$$
\lim _{n \rightarrow \infty} \operatorname{Cov}\left(y_{i_{1}, n}, y_{i_{2}, n}\right)=\lim _{n \rightarrow \infty} \mathbb{E}_{n}\left(y_{i_{1}, n} y_{i_{2}, n}\right)
$$


exists for any $1 \leq i_{1}, i_{2} \leq l$.

Then the joint distribution of the tuple

$$
\left(y_{1, n}, \ldots, y_{l, n}\right)
$$

of centered random variables converges to a Gaussian distribution in the limit when $n \rightarrow \infty$, in the weak topology of probability measures.

Proof We consider the cumulant of the random variables $y_{i_{1}, n}, \ldots, y_{i_{\ell}, n}$

$$
\kappa_{\ell}\left(y_{i_{1}, n}, \ldots, y_{i_{\ell}, n}\right):=\left.\frac{\partial^{\ell}}{\partial t_{1} \cdots \partial t_{\ell}} \log \mathbb{E}_{n}\left(e^{t_{1} y_{i_{1}, n}+\cdots+t_{\ell} y_{i_{\ell}, n}}\right)\right|_{t_{1}=\cdots=t_{\ell}=0}
$$

For $\ell=1$ this cumulant

$$
\kappa_{1}\left(y_{i_{1}, n}\right)=\mathbb{E}_{n} y_{i_{1}, n}=0
$$

trivially vanishes by the centeredness.

For $\ell \geq 2$ the cumulant is shift-invariant, thus the assumption of the approximate factorization property implies that

$$
\kappa_{\ell}\left(y_{i_{1}, n}, \ldots, y_{i_{\ell}, n}\right)=n^{\frac{\ell-\operatorname{deg} \mathbf{x}_{i_{1}}-\cdots-\operatorname{deg} \mathbf{x}_{i_{\ell}}}{2}} \kappa_{\ell}\left(X_{i_{1}, n}, \ldots, X_{i_{\ell}, n}\right)=O\left(n^{\frac{2-\ell}{2}}\right) .
$$

In particular, for $\ell \geq 3$ this cumulant clearly converges to zero.

For $\ell=2$ the cumulant coincides with the covariance

$$
\kappa_{2}\left(y_{i_{1}, n}, y_{i_{2}, n}\right)=\operatorname{Cov}\left(y_{i_{1}, n}, y_{i_{2}, n}\right)
$$

and hence by it converges by assumption.

To summarize: we proved that each of the cumulants (32) converges to a finite limit. Since each mixed moment of a collection of random variables can be expressed as a polynomial in their cumulants (for example, via the moment-cumulant formula [44]), it follows that the tuple (31) converges in moments to some distribution. The multidimensional Gaussian distribution can be characterized by the property that its cumulants vanish (except for the mean value and the covariance) thus this limit is a Gaussian distribution. The Gaussian distribution is uniquely determined by its moments; it follows that convergence in moments implies weak convergence, as required.

\section{Kerov-Olshanski algebra and its spin analogue}

The usual (linear) Kerov-Olshanski algebra $\mathbb{A}[14,23]$ (also known under the less compact name algebra of polynomial functions on the set of Young diagrams; in the monograph [29, Sect. 7] it is referred to as Ivavov-Kerov algebra) is an important tool in the (linear) asymptotic representation theory of the symmetric groups. One of its 
advantages comes from the fact that it can be characterized in several equivalent ways (for example as the algebra $\Lambda^{*}$ of shifted symmetric functions); it also has several convenient linear and algebraic bases which are related to various viewpoints and aspects of the asymptotic representation theory. In particular, it was an important ingredient in the proof of Gaussianity of fluctuations for random (non-shifted) Young diagrams [41].

For the purposes of the current paper we will need the spin analogue $\Gamma$ of KerovOlshanski algebra [16].

In the current section we shall present these two algebras, as well as their modifications related to a different multiplicative structure ("disjoint product") and the links between them. The main result of the current section is Theorem 4.2. Our strategy of proof is to use the link between the linear and the spin setup which we explored in [31]. This section is purely algebraic: all calculations are exact, there are no asymptotic assumptions, there is no randomness, there are no representations and no random Young diagrams.

\subsection{The linear setup}

\subsubsection{Normalized characters of the symmetric groups}

The usual way of viewing the characters of the symmetric groups is to fix the irreducible representation $\lambda$ and to consider the character as a function of the conjugacy class $\pi$. However, there is also another very successful viewpoint due to Kerov and Olshanski [23], called dual approach, which suggests to do roughly the opposite. It turns out that the most convenient way to pursue in this direction is to define-for a fixed integer partition $\pi$-the normalized character on the conjugacy class $\pi$ as a function on the set of all Young diagrams $\mathrm{Ch}_{\pi}: \mathbb{Y} \rightarrow \mathbb{C}$ given by

$$
\mathrm{Ch}_{\pi}(\lambda):= \begin{cases}n^{\downarrow k} \operatorname{tr} \rho^{\lambda}\left(\pi \cup 1^{n-k}\right) & \text { if } n \geq k \\ 0 & \text { otherwise }\end{cases}
$$

where $n=|\lambda|$ and $k=|\pi|$. Above, $\rho^{\lambda}\left(\pi \cup 1^{n-k}\right)$ denotes the irreducible representation of the symmetric group $\mathfrak{S}_{n}$ evaluated on any permutation from $\mathfrak{S}_{n}$ with the cycle decomposition given by the partition $\pi \cup 1^{n-k}$; furthermore $t r$ is the normalized trace (9); and $n^{\downarrow k}=n(n-1) \cdots(n-k+1)$ denotes the falling power.

\subsubsection{Kerov-Olshanski algebra}

For the purposes of the current paper, Kerov-Olshanski algebra

$$
\mathbb{A}:=\operatorname{span}\left\{\mathrm{Ch}_{\pi}: \pi \in \mathcal{P}\right\}
$$


may be defined as the linear span of the normalized linear characters of the symmetric groups. We equip if with a filtration $\mathcal{F}_{0} \subseteq \mathcal{F}_{1} \subseteq \cdots \subseteq \mathbb{A}$ given by

$$
\mathcal{F}_{k}:=\operatorname{span}\left\{\mathrm{Ch}_{\pi}: \pi \in \mathcal{P}, \quad\|\pi\| \leq k\right\}
$$

where

$$
\|\pi\|=|\pi|+\ell(\pi) .
$$

This specific choice of the filtration is motivated by investigation of asymptotics of (random) Young diagrams and tableaux in the scaling in which they grow to infinity in such a way that they remain balanced $[4,41]$.

\subsubsection{Disjoint product and the algebra $\mathbb{A}$.}

The characters $\left(\mathrm{Ch}_{\pi}: \pi \in \mathcal{P}\right)$ turn out to form a linear basis of $\mathbb{A}$ which allows us to define a new multiplication on $\mathbb{A}$, the disjoint product, by setting

$$
\mathrm{Ch}_{\pi^{1}} \bullet \mathrm{Ch}_{\pi^{2}}:=\mathrm{Ch}_{\pi^{1} \pi^{2}},
$$

where the product of two partitions $\pi^{1} \pi^{2}$ on the right-hand side should be understood-just like in Sect. 2.5.1—as their concatenation.

The vector space $\mathbb{A}$ equipped with the disjoint product $\bullet$ becomes a unital, commutative, $\mathbb{Z}_{\geq 0}$-filtered (with respect to the usual filtration (33)) algebra which will be denoted by $\overline{\mathbb{A}}_{\text {. }}$.

To summarize: the vector space $\mathbb{A}$ can be equipped with two distinct multliplicative structures which correspond to the pointwise product and to the disjoint product. Comparison of these two multiplicative structures turns out to be a fruitful idea in asymptotic representation theory [41]. We recall the key result of this flavour below in Theorem 4.1.

\subsubsection{Approximate factorization property for linear characters}

The following result turned out to be essential for proving Gaussianity of fluctuations for random Young diagrams in [41]. Our goal in this section is to prove its spin analogue (Theorem 4.2).

Proposition 4.1 The identity map

$$
\operatorname{id}_{\mathbb{A}}: \mathbb{A}_{\bullet} \longrightarrow \mathbb{A}
$$

has the approximate factorization property.

Furthermore, the second cumulant of this map is given by

$$
\kappa_{2}^{\mathrm{id}_{\mathbb{A}}}\left(\mathrm{Ch}_{k_{1}}, \mathrm{Ch}_{k_{2}}\right)=\mathrm{Ch}_{k_{1}, k_{2}}-\mathrm{Ch}_{k_{1}} \mathrm{Ch}_{k_{2}}
$$




$$
\begin{aligned}
& =(-1) \sum_{r \geq 1} \sum_{\substack{a_{1}, \ldots, a_{r} \geq 1 \\
a_{1}+\cdots+a_{r}=k_{1}}} \sum_{\substack{b_{1}, \ldots, b_{r} \geq 1 \\
b_{1}+\cdots+b_{r}=k_{2}}} \frac{k_{1} k_{2}}{r} \mathrm{Ch}_{\left(a_{1}+b_{1}-1\right), \ldots,\left(a_{r}+b_{r}-1\right)} \\
& + \text { terms of degree at most }\left(k_{1}+k_{2}-2\right) \text {. }
\end{aligned}
$$

The first proof of this result was found by the second-named author [41, Theorem 15]. For a sketch of an alternative proof based on Stanley character formula and some more historical context we refer to [42, Sect. 1.13]. An extension of this result to the context of Jack symmetric functions and Jack characters was proved by a yet another method in [43, Theorem 2.3].

\subsection{The spin setup}

\subsubsection{Normalized spin characters}

Following Ivanov [16,17] (see also [31]), for a fixed odd partition $\pi \in \mathcal{O P}$ the corresponding normalized spin character is a function on the set of all strict partitions given by

$$
\mathrm{Ch}_{\pi}^{\text {spin }}(\xi):= \begin{cases}n^{\downarrow k} 2^{\frac{\|\pi\|}{2}} \frac{\phi^{\xi}\left(\pi \cup 1^{n-k}\right)}{\phi^{\xi}\left(1^{n}\right)} & \text { if } n \geq k, \\ 0 & \text { otherwise }\end{cases}
$$

where $n=|\xi|$ and $k=|\pi|$.

\subsubsection{Spin Kerov-Olshanski algebra}

We define the spin Kerov-Olshanski algebra (maybe Ivanov algebra would be an even better name)

$$
\Gamma:=\operatorname{span}\left\{\mathrm{Ch}_{\pi}^{\text {spin }}: \pi \in \mathcal{S P}\right\}
$$

as the linear span of spin characters [16, Sect. 6]. Ivanov proved that the elements of $\Gamma$ can be identified with supersymmetric polynomials, thus $\Gamma$ is a unital, commutative algebra. Following [31, Sect. 1.6], we equip $\Gamma$ with a filtration $\mathcal{G}_{0} \subseteq \mathcal{G}_{1} \subseteq \cdots \subseteq \Gamma$ defined by

$$
\mathcal{G}_{k}:=\operatorname{span}\left\{\mathrm{Ch}_{\pi}^{\text {spin }}: \pi \in \mathcal{O P}, \quad\|\pi\| \leq k\right\}
$$

\subsubsection{Disjoint product and the algebra $\Gamma_{\bullet}$}

Similarly as in Sect. 4.1.3 we define the disjoint product of spin characters

$$
\mathrm{Ch}_{\pi^{1}}^{\text {spin }} \bullet \mathrm{Ch}_{\pi^{2}}^{\text {spin }}:=\mathrm{Ch}_{\pi^{1} \pi^{2}}^{\text {spin }}
$$


for arbitrary $\pi_{1}, \pi_{2} \in \mathcal{S P}$. We denote by $\Gamma_{\bullet}$ the filtered algebra which, as a vector space, coincides with $\Gamma$ and is equipped with a multiplication given by the disjoint product $\bullet$.

\subsubsection{Approximate factorization property for spin characters}

Theorem 4.2 The identity map

$$
\operatorname{id}_{\Gamma}: \Gamma \bullet \Gamma
$$

has the approximate factorization property.

Furthermore, the second cumulant of this map is given-for any odd integers $k_{1}, k_{2} \geq 1-b y$

$$
\begin{aligned}
\kappa_{2}^{\mathrm{id}} \Gamma & \left(\mathrm{Ch}_{k_{1}}^{\mathrm{spin}}, \mathrm{Ch}_{k_{2}}^{\mathrm{spin}}\right)=\mathrm{Ch}_{k_{1}, k_{2}}^{\mathrm{spin}}-\mathrm{Ch}_{k_{1}}^{\mathrm{spin}} \mathrm{Ch}_{k_{2}}^{\mathrm{spin}} \\
= & (-1) \sum_{r \geq 1} \frac{2^{r-1}}{r} k_{1} k_{2} \sum_{\left(a_{i}\right),\left(b_{i}\right)} \mathrm{Ch}_{\left(a_{1}+b_{1}-1\right), \ldots,\left(a_{r}+b_{r}-1\right)}^{\mathrm{spin}} \\
& + \text { terms of degree at most }\left(k_{1}+k_{2}-2\right),
\end{aligned}
$$

where the second sum runs over integers $a_{1}, \ldots, a_{r}, b_{1}, \ldots, b_{r} \geq 1$ such that $a_{1}+$ $\cdots+a_{r}=k_{1}$, and $b_{1}+\cdots+b_{r}=k_{2}$ and for each $i \in[r]$ the sum $a_{i}+b_{i}$ is even.

The proof is postponed to Sect. 4.6. Our strategy is to explore the link between the linear and the spin setup.

\subsection{Double of a function: Kerov-Olshanski algebra: linear versus spin}

\subsubsection{Double of a strict partition}

We denote by $\mathcal{P}_{n}$ the set of partitions of a given integer $n \geq 0$. The theory of partitions and Young diagrams is more developed than its shifted counterpart. For this reason it is convenient to encode a given strict partition $\xi \in \mathcal{S} \mathcal{P}_{n}$ by its double $D(\xi) \in$ $\mathcal{P}_{2 n}$. Graphically, $D(\xi)$ corresponds to a Young diagram obtained by arranging the shifted Young diagram $\xi$ and its 'transpose' so that they nicely fit along the 'diagonal', cf. Fig. 1, see also [27, p. 9].

\subsubsection{Double of a function}

If $F: \mathcal{P} \rightarrow \mathbb{C}$ is a function on the set of partitions, we define its double as the function $D^{*} F: \mathcal{S P} \rightarrow \mathbb{C}$ on the set of strict partitions

$$
\left(D^{*} F\right)(\xi):=F(D(\xi)) \quad \text { for } \xi \in \mathcal{S P}
$$

given by doubling of the argument. 
Proposition 4.3 ([31, Theorems 1.8, 1.9]) The map $D^{*}$ is an algebra homomorphism which maps Kerov-Olshanski algebra to its spin counterpart:

$$
D^{*}(\mathbb{A})=\Gamma
$$

and, additionally, preserves the filtration, i.e.

$$
\mathcal{G}_{k}=D^{*}\left(\mathcal{F}_{k}\right) \quad \text { for any integer } k \geq 0
$$

In the following we well need to compute the images $D^{*} \mathrm{Ch}_{\rho}$ of the linear basis of $\mathbb{A}$. The following two results provide the necessary information.

Proposition 4.4 ([31, Theorem 3.1]) In the case when $\rho \in \mathcal{O P}$ is an odd partition,

$$
D^{*} \mathrm{Ch}_{\rho}=\sum_{I \subseteq\{1,2, \ldots, \ell(\rho)\}} \mathrm{Ch}_{\rho(I)}^{\text {spin }} \mathrm{Ch}_{\rho\left(I^{c}\right)}^{\text {spin }} \in \Gamma
$$

where $\rho(I)=\left(\rho_{i_{1}}, \rho_{i_{2}}, \ldots, \rho_{i_{r}}\right)$ for $I=\left\{i_{1}<i_{2}<\cdots<i_{r}\right\}$ and $I^{c}=$ $\{1, \ldots, \ell(\rho)\} \backslash I$ denotes the complement of $I$.

Proposition 4.5 In the case when $\rho \notin \mathcal{O P}$ is not an odd partition, $D^{*} \mathrm{Ch}_{\rho} \in \Gamma$ is of degree at most

$$
\left\{\begin{array}{l}
\|\rho\|-1 \text { if } \rho \text { contains exactly one part which is even } \\
\|\rho \mid\|-2 \text { if } \rho \text { contains at least two parts which are even. }
\end{array}\right.
$$

Proof We start with the special case when $\rho \notin \mathcal{O P}$ contains exactly one part which is even. In this case $\|\rho\| \|$ is an odd integer.

Clearly $\mathrm{Ch}_{\rho} \in \mathcal{F}_{\|\rho\| \|}$; Eq. (41) implies therefore that $D^{*} \mathrm{Ch}_{\rho} \in \mathcal{G}_{\|\rho\|}$. We revisit the definition (38) of the filtration $\mathcal{G}$. Note that $\|\pi\|$ is always an even integer for any $\pi \in \mathcal{O P}$; it follows therefore that $\mathcal{G}_{2 k+1}=\mathcal{G}_{2 k}$ for any integer $k \geq 0$. In particular, $\mathcal{G}_{\|\rho\| \|}=\mathcal{G}_{\|\rho\| \|-1}$. In this way we proved that $D^{*} \mathrm{Ch}_{\rho} \in \mathcal{G}_{\|\rho\|-1}$, as required.

Let $\rho=\left(\rho_{1}, \ldots, \rho_{\ell}\right) \notin \mathcal{O P}$ be now a general partition. We consider the identity

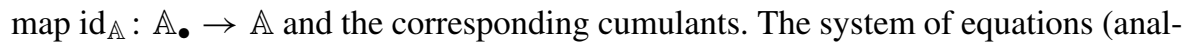
ogous to (27)) which express the cumulants in terms of the moments can be inverted. The resulting moment-cumulant formula [44] expresses any given moment

$$
\mathrm{Ch}_{\rho}=\mathrm{id}_{\mathbb{A}}\left(\mathrm{Ch}_{\rho_{1}} \bullet \cdots \bullet \mathrm{Ch}_{\rho_{\ell}}\right)
$$

as a polynomial in terms of the cumulants of the individual factors $\mathrm{Ch}_{\rho_{1}}, \ldots, \mathrm{Ch}_{\rho_{\ell}}$. For example,

$$
\begin{aligned}
\mathrm{Ch}_{\rho_{1}}=\mathrm{id}_{\mathbb{A}}\left(\mathrm{Ch}_{\rho_{1}}\right) & =\kappa_{1}^{\mathrm{id}_{\mathbb{A}}}\left(\mathrm{Ch}_{\rho_{1}}\right), \\
\mathrm{Ch}_{\rho_{1}, \rho_{2}}=\mathrm{id}_{\mathbb{A}}\left(\mathrm{Ch}_{\rho_{1}} \bullet \mathrm{Ch}_{\rho_{2}}\right) & =\kappa_{2}^{\operatorname{id}_{\mathbb{A}}}\left(\mathrm{Ch}_{\rho_{1}}, \mathrm{Ch}_{\rho_{2}}\right)+\kappa_{1}^{\mathrm{id}_{\mathbb{A}}}\left(\mathrm{Ch}_{\rho_{1}}\right) \kappa_{1}^{\mathrm{id}_{\mathbb{A}}}\left(\mathrm{Ch}_{\rho_{2}}\right) .
\end{aligned}
$$


In the general case, the summands in such an expansion of (44) can be split into the following two classes:

(a) the unique summand

$$
\kappa_{1}^{\mathrm{id}_{\mathbb{A}}}\left(\mathrm{Ch}_{\rho_{1}}\right) \cdots \kappa_{1}^{\mathrm{id}_{\mathbb{A}}}\left(\mathrm{Ch}_{\rho_{\ell}}\right)=\mathrm{Ch}_{\rho_{1}} \cdots \mathrm{Ch}_{\rho_{\ell}}
$$

(b) the remaining summands; each such a summand contains at least one factor with a cumulant $\kappa_{k} \operatorname{id}_{\mathbb{A}}$ for some $k \geq 2$.

We apply the map $D^{*}$ to (44) or, equivalently, to its aforementioned expansion to the products of cumulants and we investigate the resulting terms.

In the case (a), by applying $D^{*}$ to $(45)$ we get

$$
\left(D^{*} \mathrm{Ch}_{\rho_{1}}\right) \cdots\left(D^{*} \mathrm{Ch}_{\rho_{\ell}}\right) .
$$

The discussion from the very beginning of this proof (the special case when $\rho$ has exactly one even part) it follows that for each of the factors we have that $D^{*} \mathrm{Ch}_{\rho_{i}} \in$ $\mathcal{G}_{\rho_{i}+1}$ if $\rho_{i}$ is odd and $D^{*} \mathrm{Ch}_{\rho_{i}} \in \mathcal{G}_{\rho_{i}}$ if $\rho_{i}$ is even. Thus the degree of (46) is bounded from above by

$$
\|\rho\|-\text { (number of parts of } \rho \text { which are even). }
$$

In the case (b), by approximate factorization property each cumulant $\kappa_{k}^{\mathrm{id}_{\mathbb{A}}}$ causes a decrease of the degree by $2(k-1)$. It follows that the image of the considered summand under the map $D^{*}$ is of degree at most $\|\rho\| \|-2$.

This completes the proof.

Remark 4.6 It would be very interesting to have some explicit closed formula (maybe in the flavour of Eq. (42)) for $D^{*} \mathrm{Ch}_{\rho}$ in the case when $\rho \notin \mathcal{O P}$. Such a formula would make the link between Kerov-Olshanski algebra $\mathbb{A}$ and its spin counterpart $\Gamma$ even more explicit.

We conjecture that the degree bound (43) is not optimal and that $D^{*} \mathrm{Ch}_{\rho}$ is of degree at most

$$
\|\rho\|-\text { (number of parts in } \rho \text { which are even) }
$$

for an arbitrary partition $\rho$.

\subsection{Abstract viewpoint on Proposition 4.4}

We define the vector space

$$
\mathbb{A}_{\bullet}^{\text {odd }}:=\operatorname{span}\left\{\mathrm{Ch}_{\pi}: \pi \in \mathcal{O P}\right\} \subset \mathbb{A}
$$

which is spanned by the characters corresponding to odd partitions. This vector space, equipped with the disjoint product $\bullet$, is a unital, commutative algebra. 
We consider the algebra homomorphism $\Psi: \mathbb{A}_{\bullet}^{\text {odd }} \rightarrow \Gamma_{\bullet} \otimes \Gamma_{\bullet}$ which is defined on the algebraic basis of $\mathbb{A}_{\bullet}^{\text {odd }}$ by an analogue of the Leibniz rule

$$
\Psi\left(\mathrm{Ch}_{k}\right)=\mathrm{Ch}_{k}^{\text {spin }} \otimes 1+1 \otimes \mathrm{Ch}_{k}^{\text {spin }} \quad \text { for any odd integer } k \geq 1 .
$$

It follows that for any $\rho \in \mathcal{O P}$

$$
\Psi\left(\mathrm{Ch}_{\rho}\right)=\sum_{I \subseteq\{1,2, \ldots, \ell(\rho)\}} \mathrm{Ch}_{\rho(I)}^{\text {spin }} \otimes \mathrm{Ch}_{\rho\left(I^{c}\right)}^{\text {spin }} .
$$

The pointwise product gives rise to a bilinear function $m: \Gamma \times \Gamma \rightarrow \Gamma$ given by $m(F, G):=F G$ which can be lifted to the unique linear map $m: \Gamma \otimes \Gamma \rightarrow \Gamma$ on the tensor product. Because of the isomorphism of the vector spaces $\Gamma \cong \Gamma$ • we will view $m$ as a map $m: \Gamma_{\bullet} \otimes \Gamma_{\bullet} \rightarrow \Gamma$.

With these notations, the following result is a straightforward reformulation of Proposition 4.4.

Corollary 4.7 The following diagram commutes

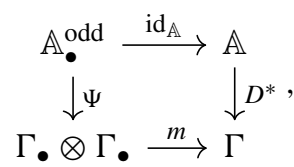

where the upper horizontal arrow is an inclusion of vector spaces.

\subsection{Cumulants of characters}

The following result provides a direct link between the cumulants for $\operatorname{id}_{\mathbb{A}}: \mathbb{A} \bullet \rightarrow \mathbb{A}$ and $\operatorname{id}_{\Gamma}: \Gamma_{\bullet} \rightarrow \Gamma$.

Theorem 4.8 For any odd integers $k_{1}, \ldots, k_{\ell} \geq 1$

$$
\kappa_{\ell}^{\mathrm{id} \Gamma}\left(\mathrm{Ch}_{k_{1}}^{\text {spin }}, \ldots, \mathrm{Ch}_{k_{\ell}}^{\text {spin }}\right)=\frac{1}{2} D^{*}\left(\kappa_{\ell}^{\mathrm{id}_{\mathbb{A}}}\left(\mathrm{Ch}_{k_{1}}, \ldots, \mathrm{Ch}_{k_{\ell}}\right)\right)
$$

where the cumulant on the left hand side concerns the identity map

$$
\operatorname{id}_{\Gamma}: \Gamma_{\bullet} \rightarrow \Gamma
$$

while the cumulant on the right-hand side concerns the identity map

$$
\operatorname{id}_{\mathbb{A}}: \mathbb{A}_{\bullet} \rightarrow \mathbb{A}
$$


Proof Since both vertical arrows in the commutative diagram (47) are unital homomorphisms of algebras, it follows immediately that the cumulants for the two horizontal arrows are related by the identity

$$
D^{*}\left(\kappa_{\ell}^{\mathrm{id}_{\mathbb{A}}}\left(x_{1}, \ldots, x_{\ell}\right)\right)=\kappa_{\ell}^{m}\left(\Psi\left(x_{1}\right), \ldots, \Psi\left(x_{\ell}\right)\right)
$$

for any $x_{1}, \ldots, x_{\ell} \in \mathbb{A}_{\bullet}^{\text {odd }}$.

We will consider the special case of this equality when each $x_{i}=\mathrm{Ch}_{k_{i}}$ is a character corresponding to a partition $\left(k_{i}\right) \in \mathcal{O P}$ which consists of a single part which is odd. Then

$$
\begin{aligned}
& D^{*}\left(\kappa_{\ell}^{\mathrm{id}_{\mathbb{A}}}\left(\mathrm{Ch}_{k_{1}}, \ldots, \mathrm{Ch}_{k_{\ell}}\right)\right) \\
& \quad=\kappa_{\ell}^{m}\left(\mathrm{Ch}_{k_{1}}^{\text {spin }} \otimes 1+1 \otimes \mathrm{Ch}_{k_{1}}^{\text {spin }}, \ldots, \mathrm{Ch}_{k_{\ell}}^{\text {spin }} \otimes 1+1 \otimes \mathrm{Ch}_{k_{\ell}}^{\text {spin }}\right) .
\end{aligned}
$$

Since the cumulant is linear with respect to each of its arguments, the right-hand side can be expanded to $2^{\ell}$ summands. Each summand is the cumulant $\kappa_{\ell}^{m}$ applied to an $\ell$-tuple of elements, with each element either from the subalgebra $\Gamma_{\bullet} \otimes 1$ or from the subalgebra $1 \otimes \Gamma_{\bullet}$. Since these two subalgebras are classically independent with respect to the expected value $m$, any mixed cumulant vanishes. It follows that out of these $2^{\ell}$ summands there are only two which are non-zero and

$$
\begin{aligned}
& \kappa_{\ell}^{m}\left(\mathrm{Ch}_{k_{1}}^{\text {spin }} \otimes 1+1 \otimes \mathrm{Ch}_{k_{1}}^{\text {spin }}, \ldots, \mathrm{Ch}_{k_{\ell}}^{\text {spin }} \otimes 1+1 \otimes \mathrm{Ch}_{k_{\ell}}^{\text {spin }}\right) \\
& \quad=\kappa_{\ell}^{m}\left(\mathrm{Ch}_{k_{1}}^{\text {spin }} \otimes 1, \ldots, \mathrm{Ch}_{k_{\ell}}^{\text {spin }} \otimes 1\right)+\kappa_{\ell}^{m}\left(1 \otimes \mathrm{Ch}_{k_{1}}^{\text {spin }}, \ldots, 1 \otimes \mathrm{Ch}_{k_{\ell}}^{\text {spin }}\right) \\
& \quad=2 \kappa_{\ell}^{\mathrm{id}}\left(\mathrm{Ch}_{k_{1}}^{\text {spin }}, \ldots, \mathrm{Ch}_{k_{\ell}}^{\text {spin }}\right) .
\end{aligned}
$$

\subsection{Proof of Theorem 4.2}

Proof of Theorem 4.2 By Lemma 3.8, in order to prove the first part it is enough to show that the cumulant on the left-hand side of (48) is an element of $\Gamma$ of degree at most

$$
\left(k_{1}+1\right)+\cdots+\left(k_{\ell}+1\right)-2(\ell-1) .
$$

However, the approximate factorization property for the map $\mathrm{id}_{\mathbb{A}}$ (Theorem 4.1) combined with Proposition 4.3 show this degree bound for the right-hand side of (48), as required. 
For the second part we apply (48) in the special case $\ell=2$. The second cumulant $\kappa_{2}^{\operatorname{id}_{\mathbb{A}}}$ which contributes to the right-hand side is explicitly given by (35). It remains now to evaluate $D^{*} \mathrm{Ch}_{\rho}$ for

$$
\rho=\left(\left(a_{1}+b_{1}-1\right), \ldots,\left(a_{r}+b_{r}-1\right)\right)
$$

A simple parity argument shows that there is an even number of even parts of such a partition $\rho$. In particular, if $\rho \notin \mathcal{O P}$ then the number of its even parts is at least 2 . Proposition 4.5 is then applicable and shows that in this case $D^{*} \mathrm{Ch}_{\rho}$ is of degree at most $k_{1}+k_{2}-2$.

In the opposite case when $\rho \in \mathcal{O P}$, the exact value of $D^{*} \mathrm{Ch}_{\rho}$ is given by Proposition 4.4. This exact form can be simplified thanks to the observation that by approximate factorization property for $\mathrm{id}_{\Gamma}$

$$
\begin{aligned}
\kappa_{2}^{\mathrm{id}} \Gamma & \left(\mathrm{Ch}_{\rho(I)}^{\text {spin }}, \mathrm{Ch}_{\rho\left(I^{c}\right)}^{\text {spin }}\right)=\mathrm{Ch}_{\rho(I)}^{\text {spin }} \bullet \mathrm{Ch}_{\rho\left(I^{c}\right)}^{\text {spin }}-\mathrm{Ch}_{\rho(I)}^{\text {spin }} \mathrm{Ch}_{\rho\left(I^{c}\right)}^{\text {spin }} \\
= & \mathrm{Ch}_{\rho}^{\text {spin }}-\mathrm{Ch}_{\rho(I)}^{\text {spin }} \mathrm{Ch}_{\rho\left(I^{c}\right)}^{\text {spin }} \in \mathcal{G}_{\|\rho\|-2} ;
\end{aligned}
$$

it follows that

$$
D^{*} \mathrm{Ch}_{\rho}=2^{\ell(\rho)} \mathrm{Ch}_{\rho}^{\text {spin }}+(\text { summands of degree at most }\|\rho\|-2) .
$$

which completes the proof.

\subsection{Free cumulants revisited}

We revisit Sect. 2.6. For an integer $n \geq 2$ and $\xi \in \mathcal{S P}$ we define

$$
\begin{aligned}
& S_{n}^{\text {spin }}(\xi)=S_{n}\left(\omega_{\xi}\right)=(n-1) \int_{\mathbb{R}} z^{n-2} \sigma_{\omega_{\xi}}(z) \mathrm{d} z, \\
& R_{n}^{\text {spin }}(\xi)=R_{n}\left(\omega_{\xi}\right) .
\end{aligned}
$$

By the symmetry of the profile $\omega_{\xi}: \mathbb{R} \rightarrow \mathbb{R}$ it follows that $S_{n}^{\text {spin }}=R_{n}^{\text {spin }}=0$ if $n$ is an odd integer. In the following we view $S_{n}^{\text {spin }}, R_{n}^{\text {spin }}: \mathcal{S P} \rightarrow \mathbb{R}$ as functions on the set of strict partitions.

Note that the free cumulants for strict partitions defined above and the ones considered by Matsumoto [28] differ by a factor of 2 .

Proposition 4.9 For each even integer $n \geq 2$ we have that $S_{n}^{\text {spin }}, R_{n}^{\text {spin }} \in \Gamma$ with $\operatorname{deg} S_{n}^{\text {spin }}=\operatorname{deg} R_{n}^{\text {spin }}=n$. Furthermore, $\left(S_{2}^{\text {spin }}, S_{4}^{\text {spin }}, \ldots\right)$ as well as $\left(R_{2}^{\mathrm{spin}}, R_{4}^{\mathrm{spin}}, \ldots\right)$ generate $\Gamma$ as a filtered algebra. 
Proof Comparison of Figs. 2 and 3 shows that the measures $\sigma_{\omega_{\xi}}$ and $\sigma_{\omega_{D(\xi)}}$ are equal, up to a translation by $\frac{1}{2}$. In particular,

$$
\begin{aligned}
& \frac{1}{n-1}\left(D^{*} S_{n}\right)(\xi)=\frac{1}{n-1} S_{n}(D(\xi))=\int_{\mathbb{R}} z^{n-2} \sigma_{\omega_{D(\xi)}}(z) \mathrm{d} z \\
& =\int_{\mathbb{R}}\left(z+\frac{1}{2}\right)^{n-2} \sigma_{\omega_{\xi}}(z) \mathrm{d} z=\sum_{\substack{0 \leq k \leq n-2, k \text { is even }}}\left(\begin{array}{c}
n-2 \\
k
\end{array}\right) \frac{1}{2^{n-k-2}(k+1)} S_{k+2}^{\text {spin }}(\xi) .
\end{aligned}
$$

The collection of such equalities over even integers $n \in\{2,4, \ldots, 2 k\}$ shows that the linear span (with rational coefficients) of the functions $D^{*} S_{2}, D^{*} S_{4}, \ldots, D^{*} S_{2 k}$ is equal to the linear span (also with rational coefficients) of the functions $S_{2}^{\text {spin }}, S_{4}^{\text {spin }}, \ldots, S_{2 k}^{\text {spin }}$. This has a twofold consequence. Firstly, $S_{2 n}^{\text {spin }} \in \Gamma$ is of degree $2 n$, as required. Secondly, $\left(S_{2}^{\text {spin }}, S_{4}^{\text {spin }}, \ldots\right)$ generate $\Gamma$ as a filtered algebra, as required.

The systems of Eqs. (22) and (23) imply that the analogous claims hold as well for the free cumulants $R_{2}^{\text {spin }}, R_{4}^{\text {spin }}, \ldots$.

\section{The key tool}

Suppose that we are given a (sequence of) spin representation(s) and the corresponding (sequence of) character ratio(s). As we already mentioned, sometimes it is more convenient to pass from the character ratio to the corresponding cumulants. Interestingly, there are three distinct natural types of such cumulants, each having its own advantages. In the current section we will review them and prove the key tool of the current paper, Theorem 5.1 which provides a link between them.

\subsection{Three types of cumulants for the character ratios}

We will use the setup considered in Sect. 3.6, i.e. $\left(V^{(n)}\right)$ is a sequence of representations and $\left(\chi^{(n)}\right)$ is the corresponding sequence of the character ratios.

\subsubsection{Cumulants of partitions}

The first type of cumulants we will use are the ones which correspond to the linear map $\chi: \mathbb{C}[\mathcal{O P}] \rightarrow \mathcal{X}$, see Eq. (30). These cumulants $\kappa_{\ell}^{\chi}\left(\pi_{1}, \ldots, \pi_{\ell}\right)$ are indexed by odd partitions $\pi_{1}, \ldots, \pi_{\ell} \in \mathcal{O P}$.

The advantage of these cumulants lies in the observation that in the applications we are often given a representation in terms of its characters and thus such cumulants can be often calculated explicitly without much effort. Regretfully, these cumulants do not have a truly probabilistic interpretation. This kind of cumulants will appear in Theorem 5.1 within conditions (a) and (b). 


\subsubsection{Cumulants in $\Gamma$}

Let us fix for a moment an integer $n \geq 1$. We consider the discrete probability space $\Omega_{n}=\mathcal{S P} \mathcal{P}_{n}$ equipped with the probability distribution $\mathbb{P}^{V^{(n)}}$; we denote by $\xi^{(n)}$ a random strict partition in $\mathcal{S P} \mathcal{P}_{n}$ with the same probability distribution $\mathbb{P}^{(n)}$. By restricting the domain of the functions, any element $X \in \Gamma$ can be viewed as a function on the set $\mathcal{S P} \mathcal{P}_{n}$ or, equivalently, as a random variable on the probability space $\Omega_{n}$. We are interested in its expected value $\mathbb{E}^{(n)} X=\mathbb{E} X\left(\xi^{(n)}\right)$.

We define a unital map $\mathbb{E}_{\Gamma}: \Gamma \rightarrow \mathcal{X}$ by setting

$$
\mathbb{E}_{\Gamma} X=\left(\mathbb{E}^{(1)} X, \mathbb{E}^{(2)} X, \ldots\right)
$$

for any $X \in \Gamma$. In the case when $X$ is a normalized spin character, this definition takes the following more concrete form

$$
\mathbb{E}_{\Gamma}\left(\mathrm{Ch}_{\pi}^{\text {spin }}\right)=\left(x_{1}, x_{2}, \ldots\right),
$$

where

$$
x_{n}=\mathbb{E} \mathrm{Ch}_{\pi}\left(\xi^{(n)}\right)=n^{\downarrow|\pi|} 2^{\frac{\|\pi\|}{2}} \chi^{(n)}(\pi) .
$$

The last equality is a consequence of the definition (36) of the normalized spin characters.

The cumulants $\kappa_{\ell}^{\mathbb{E}_{\Gamma}}\left(X_{1}, \ldots, X_{\ell}\right)$ which correspond to this map have a direct probabilistic meaning. This kind of cumulants will appear in Theorem 5.1 within conditions (c) and (d).

\subsubsection{Cumulants in $\Gamma$.}

The equality $\Gamma=\Gamma$. between the vector spaces allows us to view the aforementioned map $\mathbb{E}_{\Gamma}$ as a function on $\Gamma_{\bullet}$. We will denote it by $\mathbb{E}_{\Gamma_{\bullet}}: \Gamma_{\bullet} \rightarrow \mathcal{X}$. The cumulants $\kappa_{\ell}^{\mathbb{E}_{\Gamma}} \cdot\left(X_{1}, \ldots, X_{\ell}\right)$ which correspond to this map do not have a probabilistic meaning. This kind of cumulants will appear in Theorem 5.1 within conditions (e) and (f).

Since the algebras $\Gamma$ and $\Gamma_{\bullet}$ have different multiplicative structures, the cumulants for the maps $\mathbb{E}_{\Gamma}$ and $\mathbb{E}_{\Gamma \text {. }}$ are also different.

\subsection{The key tool}

The following theorem is a direct analogue of a result of the second-named author [41, Theorem and Definition 1] which concerns the usual (non-projective) representations of the symmetric groups, see also [11, Theorem 2.3] for a generalization to Jack characters.

In the following for an integer (or half-integer) $k$ we denote by $n^{k}:=\left(1^{k}, 2^{k}, \ldots\right) \in$ $\mathcal{X}$ the sequence of powers of the integers. 
Theorem 5.1 Assume that for each integer $n \geq 1$ we are given a representation $V^{(n)}$ of $\mathbb{C S}_{n}^{-}$.

Then the following conditions are equivalent:

(a) for all odd partitions $\pi_{1}=\left(k_{1}\right), \ldots, \pi_{\ell}=\left(k_{\ell}\right) \in \mathcal{O P}$ which consist of exactly one part

$$
\kappa_{\ell}^{\chi}\left(\pi_{1}, \ldots, \pi_{\ell}\right) n^{\frac{\left\|\pi_{1}\right\|+\cdots+\left\|\pi_{\ell}\right\|+2(\ell-1)}{2}}=O(1)
$$

(b) for all odd partitions $\pi_{1}, \ldots, \pi_{\ell} \in \mathcal{O P}$

$$
\kappa_{\ell}^{\chi}\left(\pi_{1}, \ldots, \pi_{\ell}\right) n^{\frac{\left\|\pi_{1}\right\|+\cdots+\left\|\pi_{\ell}\right\|+2(\ell-1)}{2}}=O(1)
$$

(c) there exists a set $X \subseteq \Gamma$ which generates $\Gamma$ as a filtered algebra with the property that for all $x_{1}, \ldots, x_{\ell} \in X$

$$
\kappa_{\ell}^{\mathbb{E}_{\Gamma}}\left(x_{1}, \ldots, x_{\ell}\right) n^{-\frac{\operatorname{deg} x_{1}+\cdots+\operatorname{deg} x_{l}-2(\ell-1)}{2}}=O(1),
$$

(d) for all $x_{1}, \ldots, x_{\ell} \in \Gamma$

$$
\kappa_{\ell}^{\mathbb{E}_{\Gamma}}\left(x_{1}, \ldots, x_{\ell}\right) n^{-\frac{\operatorname{deg} x_{1}+\cdots+\operatorname{deg} x_{\ell}-2(\ell-1)}{2}}=O(1),
$$

(e) there exists a set $X \subseteq \Gamma_{\bullet}$ which generates $\Gamma_{\bullet}$ as a filtered algebra with the property that for all $x_{1}, \ldots, x_{\ell} \in X$

$$
\kappa_{\ell}^{\mathbb{E}_{\Gamma}}\left(x_{1}, \ldots, x_{\ell}\right) n^{-\frac{\operatorname{deg} x_{1}+\cdots+\operatorname{deg} x_{\ell}-2(\ell-1)}{2}}=O(1),
$$

(f) for all $x_{1}, \ldots, x_{\ell} \in \Gamma$

$$
\kappa_{\ell}^{\mathbb{E}_{\Gamma}}\left(x_{1}, \ldots, x_{\ell}\right) n^{-\frac{\operatorname{deg} x_{1}+\cdots+\operatorname{deg} x_{\ell}-2(\ell-1)}{2}}=O(1),
$$

Furthermore, if the left-hand side of any of the expressions above has a limit for $n \rightarrow$ $\infty$ for all $\ell \leq 2$ and all prescribed choices of $\pi_{1}, \ldots, \pi_{\ell}$ (respectively, $x_{1}, \ldots, x_{\ell}$ ), then each of the the left-hand sides of the above expressions has a limit for $n \rightarrow \infty$. These limits for $\ell \leq 2$ are interrelated as follows: for any odd integers $k, k_{1}, k_{2} \geq 1$

$$
\begin{aligned}
\boldsymbol{r}_{k+1} & :=\lim _{n \rightarrow \infty} n^{\frac{k-1}{2}} \chi^{(n)}(k) \\
& =\lim _{n \rightarrow \infty} n^{\frac{k-1}{2}} \mathbb{E} \chi^{\xi^{(n)}}(k) \\
& =\lim _{n \rightarrow \infty}(2 n)^{-\frac{k+1}{2}} \cdot 2 \cdot \mathbb{E C h}_{k}^{\text {spin }}\left(\xi^{(n)}\right) \\
& =\lim _{n \rightarrow \infty}(2 n)^{-\frac{k+1}{2}} \mathbb{E} R_{k+1}^{\text {spin }}\left(\xi^{(n)}\right), \\
\boldsymbol{k}_{k_{1}+1, k_{2}+1} & :=\lim _{n \rightarrow \infty} n^{\frac{k_{1}+k_{2}}{2}} \cdot 2 \cdot\left(\chi^{(n)}\left(k_{1}, k_{2}\right)-\chi^{(n)}\left(k_{1}\right) \chi^{(n)}\left(k_{2}\right)\right),
\end{aligned}
$$




$$
\begin{aligned}
\boldsymbol{k}_{k_{1}+1, k_{2}+1} & :=\lim _{n \rightarrow \infty} n^{\frac{k_{1}+k_{2}}{2}} \cdot 2 \cdot \operatorname{Cov}\left(\chi^{\xi^{(n)}}\left(k_{1}\right), \chi^{\xi^{(n)}}\left(k_{2}\right)\right) \\
& =\lim _{n \rightarrow \infty}(2 n)^{-\frac{k_{1}+k_{2}}{2}} \operatorname{Cov}\left(R_{k_{1}+1}^{\text {spin }}\left(\xi^{(n)}\right), R_{k_{2}+1}^{\text {spin }}\left(\xi^{(n)}\right)\right),
\end{aligned}
$$

where $\xi^{(n)}$ denotes a random strict partition with the probability distribution $\mathbb{P}^{(n)}$,

$$
\begin{aligned}
\boldsymbol{k}_{k_{1}+1, k_{2}+1}= & \boldsymbol{k}_{k_{1}+1, k_{2}+1}-2 k_{1} k_{2} \boldsymbol{r}_{k_{1}+1} \boldsymbol{r}_{k_{2}+1} \\
& +2 \sum_{r \geq 1} \frac{k_{1} k_{2}}{r} \sum_{\left(a_{i}\right),\left(b_{i}\right)} \boldsymbol{r}_{a_{1}+b_{1}} \cdots \boldsymbol{r}_{a_{r}+b_{r}}
\end{aligned}
$$

where the last sum runs over integers $a_{1}, \ldots, a_{r}, b_{1}, \ldots, b_{r} \geq 1$ such that $a_{1}+\cdots+$ $a_{r}=k_{1}$, and $b_{1}+\cdots+b_{r}=k_{2}$ and for each $i \in[r]$ the sum $a_{i}+b_{i}$ is even.

Proof The proof is fully analogous to [41, Theorem and Definition 1, Theorem 3]; we just have to make sure that all ingredients of the old proof have their spin counterparts. The list of new ingredients: the information about disjoint cumulants is provided by Theorem 4.2, the link between non-normalized and normalized spin characters is provided by definition (36). The calculation is fully analogous to the linear case, however one should carefully track the powers of 2 which come from two distinct sources: the choice of normalization used in (36) as well as (39).

\subsection{Proof of Theorem 2.5}

Proof of Theorem 2.5 The proof of a very similar result presented in [11, Sect. 5] works also in our setup. In fact, we consider the simplest possible case of the latter result (with the notations of [11] this corresponds to $\alpha=1, g=0$ ). We just have to make sure that all ingredients of the proof have their spin counterparts and indeed Theorem 5.1 provides the necessary tools.

Alternatively, it was observed by De Stavola [10, Sect. 4.5] that central limit theorem (a la Theorem 2.6) for shifted Young diagrams implies also law of large numbers. The proof of De Stavola can be adapted easily to our context.

\subsection{Proof of Theorem 2.6}

Proof of Theorem 2.6 This proof is fully analogous to the proof of [41, Corollary 4]: in order to show convergence to the Gaussian distribution it is enough to check that the higher cumulants converge to zero. 


\section{Applications and examples}

\subsection{Example: shifted Plancherel measure}

We continue the discussion of the spin part of the left-regular representation from Example 2.4. Theorem 2.5 is applicable in this context with

$$
\begin{aligned}
\mathbf{r}_{k} & = \begin{cases}1 & \text { if } k=2, \\
0 & \text { if } k \neq 2,\end{cases} \\
\mathbf{k}_{k, l}^{\bullet} & =0
\end{aligned}
$$

for any even integers $k, l \geq 2$.

The limit shape $\Omega$ with the corresponding sequence of free cumulants given by (26) is uniquely determined to the Logan-Shepp \& Vershik-Kerov curve, see [4, example on pp. 133-134]. In this way we recover the result of De Stavola [10, Sect. 4.5].

\subsection{Example: Schur-Weyl measure}

\subsubsection{Proof of Theorem 1.3}

Proof of Theorem 1.3 We consider the vector space

$$
V_{d, n}^{\mathrm{SW}}=\left(\mathbb{C}^{d} \oplus \mathbb{C}^{d}\right)^{\otimes n}
$$

which with the action of the spin symmetric group $\widetilde{\mathfrak{S}}_{n}$ given by $[50$, Sect. 4.3 combined with (2.5)] becomes a (reducible) spin superrepresentation.

Its decomposition into irreducible components is provided by Sergeev duality [50, Theorem 4.8]; in particular it follows that the corresponding probability measure $\mathbb{P}_{d, n}^{S W}$ on $\mathcal{S P}_{n}$ coincides with the measure $\mathbb{P}_{n, d}^{\mathrm{SW}}$ considered in (7).

The character of $V_{d, n}^{\mathrm{SW}}$ is given by

$$
\chi_{d, n}^{\mathrm{SW}}(\pi)=\frac{1}{(\sqrt{2} d)^{\|\pi\|}}
$$

for any $\pi \in \mathcal{O} \mathcal{P}_{n}$. In the same way as in Example 2.4 we calculate the corresponding cumulants; it follows that

$$
\kappa_{\ell}\left(\pi_{1}, \ldots, \pi_{\ell}\right)=0
$$

for all $\ell \geq 2$.

We set $d=d_{n}$; it follows that Theorem 2.5 is applicable with

$$
\mathbf{r}_{k+1}=\left(\frac{c}{\sqrt{2}}\right)^{k-1}
$$




$$
\mathbf{k}_{k_{1}+1, k_{2}+1}^{\bullet}=0
$$

for any odd integers $k, k_{1}, k_{2} \geq 1$.

The explicit form of the limit shape $\Omega_{c}^{\mathrm{SW}}$ will be found in Sect. 7.3.

\subsubsection{Proof of Corollary 1.5}

Proof of Corollary 1.5 We are interested in the rescaled diagram $\omega_{\frac{1}{d_{n}} \xi}$, where $\xi:=$ $(Q(\mathbf{w}))_{\leq \alpha d_{n}^{2}}$ is the level curve of the recording tableau. The distribution of the random shifted Young diagram $\xi$ is given by Schur-Weyl measure $\mathbb{P}_{\left\lfloor\alpha d_{n}^{2}\right\rfloor, d}^{S W}$. It follows that Theorem 1.3 is applicable with

$$
c:=\lim _{n \rightarrow \infty} \frac{\sqrt{\left\lfloor\alpha d_{n}^{2}\right\rfloor}}{d_{n}}=\sqrt{\alpha}
$$

and that the convergence

$$
\omega \frac{1}{\sqrt{2\left\lfloor\alpha d_{n}^{2}\right\rfloor}} \xi \longrightarrow \Omega_{\sqrt{\alpha}}^{\mathrm{SW}}
$$

in the usual sense holds true.

By comparing the normalization factors it follows that $\omega_{\frac{1}{d_{n}} \xi}$ is a dilation of $\omega \frac{1}{\sqrt{2\left\lfloor\alpha d_{n}^{2}\right\rfloor}} \xi$ by the factor $\frac{\sqrt{2\left\lfloor\alpha d_{n}^{2}\right\rfloor}}{d_{n}} \rightarrow \sqrt{2 \alpha}$. It follows that the theorem holds for $\Omega_{\alpha}^{\mathcal{Q}}$ equal to the dilation of $\Omega_{\sqrt{\alpha}}^{\mathrm{SW}}$ by the factor $\sqrt{2 \alpha}$ :

$$
\Omega_{\alpha}^{\mathcal{Q}}(z)=\sqrt{2 \alpha} \Omega_{\sqrt{\alpha}}^{\mathrm{SW}}\left(\frac{z}{\sqrt{2 \alpha}}\right)
$$

The limit shape $\mathcal{Q}$ can be then recovered from the family of curves $\Omega_{\alpha}^{\mathcal{Q}}$ by setting

$$
\mathcal{Q}(x, y)=\sup \left\{\alpha \geq 0: \Omega_{\alpha}^{\mathcal{Q}}(x-y) \leq x+y\right\} .
$$

\subsubsection{Proof of Corollary 1.4}

Proof of Corollary 1.4 The level curve $\xi=(P(\mathbf{w}))_{\leq \alpha \frac{n}{d}}$ can be obtained by the following two-step procedure: firstly, we remove from the random word $\mathbf{w}$ all entries which are bigger than the threshold $\alpha \frac{n}{d}$ and denote the resulting word by $\mathbf{w}^{\prime}$; then we consider the common shape of the tableaux associated by shifted RSK correspondence to $\mathbf{w}^{\prime}$. 
We denote by $n^{\prime}$ the random length of the word $\mathbf{w}^{\prime}$. Since the distribution of $n^{\prime}$ is $\operatorname{Bin}\left(n, \alpha \frac{n}{d^{2}}\right)$ it follows that

$$
\frac{n^{\prime}}{\alpha \frac{n^{2}}{d^{2}}} \longrightarrow 1
$$

holds true in probability.

The limit shape of $\omega \frac{1}{\sqrt{2 n^{\prime}}} \xi$ is provided by Theorem 1.3 with

$$
c=\lim _{n \rightarrow \infty} \frac{\sqrt{n^{\prime}}}{\left\lfloor\alpha \frac{n}{d}\right\rfloor}=\frac{1}{\sqrt{\alpha}} ;
$$

thus

$$
\omega_{\frac{1}{\sqrt{2 n^{\prime}}} \xi} \longrightarrow \Omega_{\frac{1}{\sqrt{\alpha}}}^{\mathrm{SW}} .
$$

By comparison of the normalization factors it follows that $\omega_{\frac{d}{n} \xi}$ is a dilation of $\omega_{\frac{1}{\sqrt{2 n^{\prime}}} \xi}$ by the factor $\frac{d \sqrt{2 n^{\prime}}}{n} \rightarrow \sqrt{2 \alpha}$. It follows that the theorem holds for $\Omega_{\alpha}^{\mathcal{P}}$ equal to the dilation of $\Omega_{\frac{1}{\sqrt{\alpha}}}^{\mathrm{SW}}$ by the factor $\sqrt{2 \alpha}$ :

$$
\Omega_{\alpha}^{\mathcal{P}}(z)=\sqrt{2 \alpha} \Omega_{\frac{1}{\sqrt{\alpha}}}^{\mathrm{SW}}\left(\frac{z}{\sqrt{2 \alpha}}\right) .
$$

The limit shape $\mathcal{P}$ can be then recovered from the family of curves $\Omega_{\alpha}^{\mathcal{P}}$ by setting

$$
\mathcal{P}(x, y)=\sup \left\{\alpha \geq 0: \Omega_{\alpha}^{\mathcal{P}}(x-y) \leq x+y\right\} .
$$

\subsection{Random shifted tableaux with prescribed shape: Proof of Theorem 1.1.}

The current section is devoted to the proof of Theorem 1.1. For Reader's convenience the proof is split into several subsections. Note that some of the intermediate results might be interesting by themselves.

\subsubsection{Random shifted tableaux and the spin representation theory}

We start with the link between random shifted standard tableaux and the spin representation theory of the symmetric groups. This link (Proposition 6.1) is fully analogous to the classical link between non-shifted standard tableaux and the linear representation theory of the symmetric groups. Unfortunately, the proof of Proposition 6.1 is more complex than its classical counterpart because not all edges in the Bratteli diagram are simple. We present the details below. 


\subsubsection{Random shifted tableaux}

For a given shifted standard tableau $T$ with shape $\mu \in \mathcal{S P}_{n}$ and an integer $0 \leq m \leq n$ we denote by $T_{\leq m}$ the set of boxes of $T$ which are smaller or equal than $m$. This set of boxes corresponds to some $\xi \in \mathcal{S P}_{m}$. Thus $T_{\leq m}:=\xi$ can be regarded as a shifted partition.

We fix $\mu \in \mathcal{S P}_{n}$ and consider a uniformly random tableau $T \in \mathcal{T}_{\mu}$ with this prescribed shape. We are interested in the probability distribution of the random strict partition $T_{\leq m}$.

\subsubsection{Restriction of irreducible representations}

We fix $\mu \in \mathcal{S P}_{n}$ and consider the corresponding irreducible representation $V^{\mu}$ of $\mathbb{C S}_{n}^{-}$. For an integer $0 \leq m \leq n$ the restriction

$$
V^{\mu} \mid \begin{aligned}
& \mathbb{C} \mathfrak{S}_{n}^{-} \\
& \mathbb{C} \mathfrak{S}_{m}^{-}
\end{aligned}
$$

is a representation of the spin group algebra $\mathbb{C S}_{m}^{-}$. We are interested in the corresponding probability measure

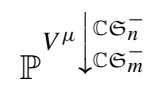

on the set $\mathcal{S P}_{m}$ which gives the probability distribution of a random irreducible component of (49).

\subsubsection{The link}

The following result shows that the two probability measures on $\mathcal{S P}_{m}$ which we considered in Sects. 6.3.2 and 6.3.3 are equal.

Proposition 6.1 Let $\mu \in \mathcal{S P}_{n}$ and $0 \leq m \leq n$. Then for any $\xi \in \mathcal{S P}_{m}$

$$
\mathbb{P}_{\mathcal{T}_{\mu}}\left(T \in \mathcal{T}_{\mu}: T_{\leq m}=\xi\right)=\left.\mathbb{P}^{V^{\mu} \mid}\right|_{\mathbb{C} \mathfrak{S}_{m}^{-}} ^{\mathbb{C}}(\xi)
$$

Proof We consider the Bratteli diagram for the sequence of superalgebras $\mathbb{C S}_{0}^{-} \subseteq$ $\mathbb{C S}_{1}^{-} \subseteq \cdots$. The vertices of this graph correspond to irreducible superrepresentations of $\mathbb{C S}_{n}^{-}$over $n \geq 0$ and hence can be identified with the strict partitions. This kind of graph (with different multiplicities of the edges) is known in the literature under the name of Schur graph [5,16].

We connect a superrepresentation $\xi$ of $\mathbb{C S}_{n-1}^{-}$by an oriented edge with a superrepresentation $\zeta$ of $\mathbb{C S}_{n}^{-}$if $\xi$ appears in the decomposition of the restriction $\zeta \downarrow_{\mathbb{C S}_{n-1}^{-}}^{\mathbb{C S}_{-}^{-}}$ into irreducible components. We declare the multiplicity of the edge to be equal to the 


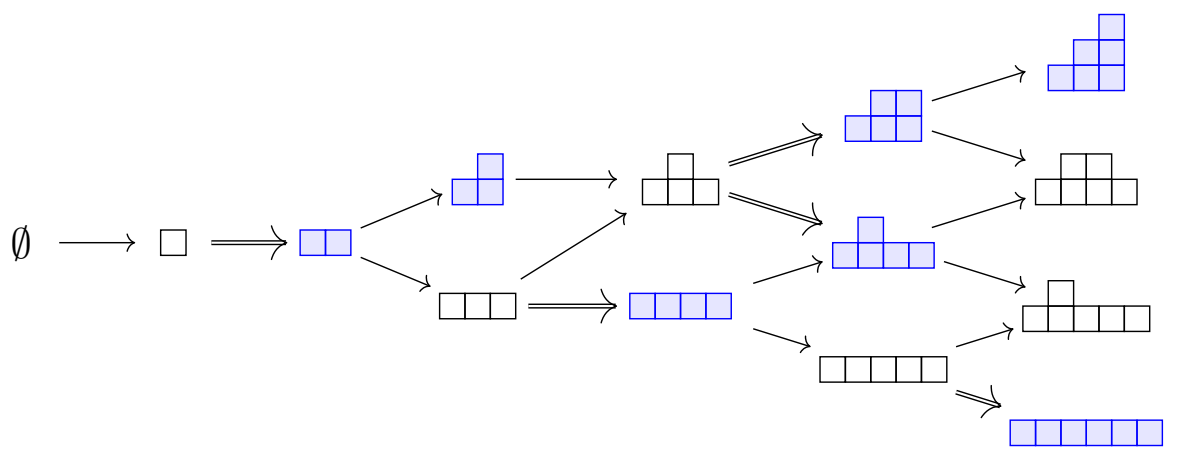

Fig. 9 Part of the Bratteli diagram for superrepresentations of spin group alegbras $\mathbb{C S}_{n}^{-}$. Shaded diagrams correspond to the elements of $\mathcal{S P}^{-}$[which correspond to the superrepresentations of type Q] while nonshaded diagrams correspond to the elements of $\mathcal{S P}^{+}$[which correspond to the superrepresentations of type M]. Double edges are drawn only on the edges from the elements of $\mathcal{S P}^{+}$to the elements of $\mathcal{S P}^{-}$; the remaining edges are simple

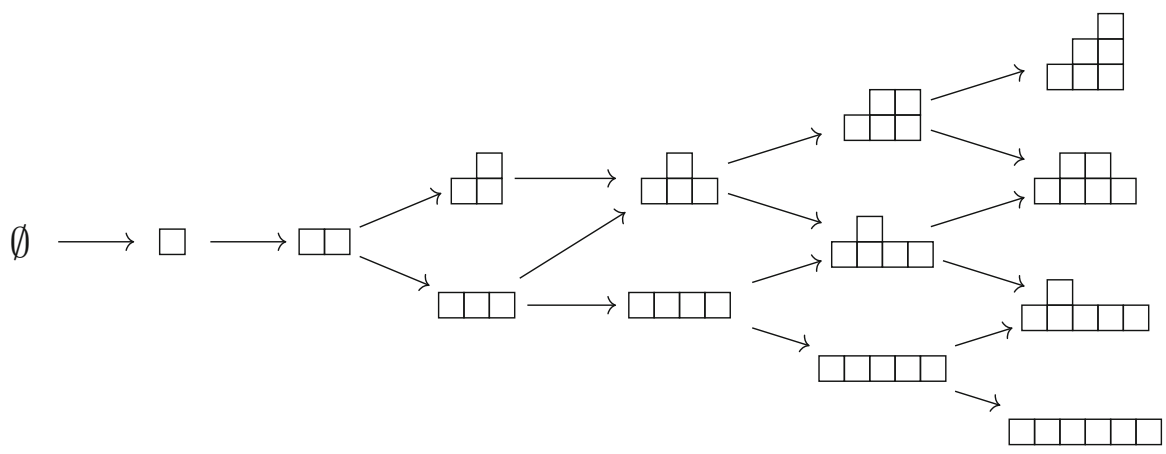

Fig. 10 Part of the shifted Young graph. This graph is obtained from Bratteli diagram (Fig. 9) by removing multiplicities from the edges

multiplicity of $\xi$ in $\zeta \downarrow_{\mathbb{C S}_{n-1}^{-}} \mathbb{C S}_{-}^{-}$. In the following we will describe the structure of this graph based on [22, Theorem 22.3.4].

For strict partitions $\xi, \zeta$ we write $\xi \nearrow \zeta$ if the shifted Young diagram $\xi$ is obtained from $\zeta$ by removal of a single box. It turns out that in the Bratteli diagram there is an oriented edge from $\xi$ to $\zeta$ if and only if $\xi \nearrow \zeta$. If this is the case, the multiplicity of this edge is given by

$$
m(\xi, \zeta)= \begin{cases}2 & \text { if } \xi \in \mathcal{S P}^{+} \text {and } \zeta \in \mathcal{S P}^{-} \\ 1 & \text { otherwise }\end{cases}
$$

see Fig. 9.

We start by pointing out that the statement of Proposition 6.1 is not very precise in the case when $\mu \in \mathcal{S P}^{-}$since in this case there are two irreducible representations which correspond to $\mu$. However, in the light of Sect. 2.4 this subtlety is irrelevant because both representations have equal character ratios on $\mathcal{O P}$. In the following we 
change our setup to superrepresentations of the superalgebras $\mathbb{C S}_{n}^{-}$and $\mathbb{C S}_{m}^{-}$; by the same argument this does not change the probability distribution on the right-hand side of (50).

Our strategy is to evaluate the denominator and the numerator on the right-hand

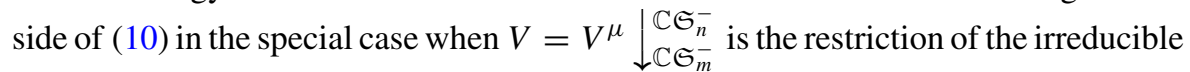
superrepresentation corresponding to $\mu$.

The denominator, the dimension of the irreducible superrepresentation $V^{\mu}$

$$
\operatorname{dim} V^{\mu}=\sum_{\emptyset=\mu^{0} \nearrow \cdots \nearrow \mu^{n}=\mu} m\left(\mu^{0}, \mu^{1}\right) \cdots m\left(\mu^{n-1}, \mu^{n}\right)
$$

is equal to the sum over all paths in the Bratteli diagram which connect the trivial onedimensional representation $\emptyset$ with $\mu$. The weight of each path is equal to the product of the multiplicities of all edges. This product is a power of 2 with the exponent $c$ equal to the number of the elements in the set

$$
\left\{0 \leq i \leq n-1:\left\|\mu^{i}\right\| \text { is even and }\left\|\mu^{i+1}\right\| \text { is odd }\right\}
$$

Since

$$
\left\|\mu^{0}\right\|, \ldots,\left\|\mu^{n}\right\|
$$

is a weakly increasing sequence of integers which at each step increases by at most one, it follows that the cardinality of (52) is equal to the number of even integers in the interval $\{0, \ldots,\|\mu\|-1\}$ which is equal to

$$
c=c_{\mu}=1+\left\lfloor\frac{\|\mu\|-1}{2}\right\rfloor .
$$

Note that this number does not depend on the choice of the path in the Bratteli diagram with a specified endpoint $\mu$. In this way we proved that

$$
\operatorname{dim} V^{\mu}=2^{c_{\mu}} \cdot\left(\text { number of paths } \emptyset=\mu^{0} \nearrow \cdots \nearrow \mu^{n}=\mu\right)
$$

The latter formula does not involve the multiplicities of the edges in the Bratteli diagram; for this reason it is convenient to replace the latter by Schur graph, see Fig. 10. The shifted Young diagram is obtained from Bratteli diagram by removing the multiplicities of the edges. Clearly, there is a bijective correspondence between paths in the shifted Young graph and shifted standard Young tableaux. In this way we proved that

$$
\operatorname{dim} V^{\mu}=2^{c_{\mu}} \cdot\left|\mathcal{T}_{\mu}\right|
$$


The numerator on the right-hand side of (10), the product

$$
n_{\xi} \operatorname{dim} V^{\xi}=\sum_{\substack{\emptyset=\mu^{0} \nearrow \ldots \nearrow \mu^{n}=\mu, \mu^{m}=\xi}} m\left(\mu^{0}, \mu^{1}\right) \cdots m\left(\mu^{n-1}, \mu^{n}\right)
$$

is a sum analogous to the right-hand side of (51) over paths which pass through the vertex $\xi$. A reasoning analogous to the one above implies that

$n_{\xi} \operatorname{dim} V^{\xi}=2^{c_{\mu}} \cdot\left(\right.$ number of paths $\emptyset=\mu^{0} \nearrow \cdots \nearrow \mu^{n}=\mu$ such that $\left.\mu^{m}=\xi\right)$.

The paths which contribute to the second factor on the right-hand side are in a bijective correspondence with the shifted tableaux

$$
\left\{T \in \mathcal{T}_{\mu}: T_{\leq m}=\xi\right\}
$$

The latter observation combined with (53) concludes the proof.

\subsubsection{Irreducible representations have approximate factorization property}

We will show that a sequence of irreducible representations $\left(V^{\xi^{(k)}}\right)$ has approximate factorization property (Definition 2.3), provided that the strict partitions $\xi^{(k)}$ converge to some limit shape. It might seem silly to prove this kind of result since the main purpose of approximate factorization property is to serve as the assumption of Theorems 2.5 and 2.6 which are trivially satisfied for irreducible representations. The key point is that Theorem 5.1 will allow us to produce new examples (often more interesting ones) of the sequences with approximate factorization property from old (and boring) examples.

Clearly, Definition 2.3 still makes sense and Theorem 5.1 remains true if we replace the sequence $\left(V^{(n)}\right)$ of representations by its subsequence $\left(V^{\left(n_{k}\right)}\right)$ for some sequence $\left(n_{k}\right)$ of positive integers which tends to infinity; in the following we will use Theorem 5.1 in this formulation.

Proposition 6.2 We keep the notations and the assumptions from Theorem 1.1. We define $V^{\left(n_{k}\right)}:=V^{\xi^{(k)}}$ to be the irreducible representation of $\mathbb{C S}_{n_{k}}^{-}$. Then the sequence $\left(V^{\xi^{(k)}}\right)$ has the approximate factorization property.

Proof The corresponding probability measure $\mathbb{P}^{V^{\left(n_{k}\right)}}$ is the point measure supported in $\xi^{(k)}$. With the notations of Sect. 5.1.2 the corresponding random strict partition is deterministic, almost surely equal to $\xi^{(k)}$. All higher cumulants $\kappa_{l}$ (with $l \geq 2$ ) of deterministic random variables are equal to zero; it follows that for $l \geq 2$ condition (c) of Theorem 5.1 is trivially fulfilled for any choice of $X \subseteq \Gamma$.

Take $X=\left\{S_{2}^{\text {spin }}, S_{4}^{\text {spin }}, \ldots\right\} \subseteq \Gamma$; our plan is to verify that condition (c) of Theorem 5.1 is fulfilled also for $l=1$. Firstly, note that by Proposition 4.9 the set $X$ indeed 
generates $\Gamma$ as a filtered algebra. The first cumulant $\kappa_{1}$ takes a particularly simple form and

$$
\kappa_{1}^{\mathbb{E}_{\Gamma}}\left(S_{2 j}^{\text {spin }}\right)=\mathbb{E}_{\Gamma}\left(S_{2 j}^{\text {spin }}\right)=\left(\mathbb{E}^{\left(n_{k}\right)} S_{2 j}^{\text {spin }}\right)_{k \geq 1}=\left(S_{2 j}^{\text {spin }}\left(\xi^{(k)}\right)\right)_{k \geq 1}
$$

The balancedness condition implies that the limit

$$
\lim _{k \rightarrow \infty} \frac{1}{n_{k}^{j}} S_{2 j}^{\text {spin }}\left(\xi^{(k)}\right)=S_{2 j}\left(\Omega_{1}\right)
$$

exists thus condition (c) of Theorem 5.1 is indeed fulfilled.

\subsubsection{Restriction of representations with approximate factorization property}

The following reasoning is based on its non-shifted analogue [41, Theorem 8].

We fix $0<\alpha<1$. We define $n_{k}^{\prime}:=\left\lfloor\alpha n_{k}\right\rfloor$ and consider a restriction

$$
W^{\left(n_{k}^{\prime}\right)}:=V^{\xi^{(k)}} \downarrow_{\mathbb{C S}_{n_{k}^{\prime}}^{-}}^{\mathbb{C S}_{n_{k}}^{-}}
$$

which is a representation of $\mathbb{C S}_{n_{k}^{\prime}}^{-}$. We claim that the sequence $\left(W^{\left(n_{k}^{\prime}\right)}\right)$ also has approximate factorization property. Indeed, in order to verify approximate factorization property (no matter if we consider $\left(V^{\xi^{(k)}}\right)$ or the sequence of its restrictions $\left(W^{\left(n_{k}^{\prime}\right)}\right)$ ) we need to understand the asymptotics of the left-hand side of (18). The only difference that in the context of $\left(V^{\xi^{(k)}}\right)$ the right-hand side is

$$
O\left(n_{k}^{-\frac{\left\|\pi^{1}\right\|+\cdots+\left\|\pi^{\ell}\right\|+2(\ell-1)}{2}}\right)
$$

while in the context of $\left.\left(W^{\left(n_{k}^{\prime}\right)}\right)\right)$ this right-hand side should be understood as

$$
O\left(\left(n_{k}^{\prime}\right)^{-\frac{\left\|\pi^{1}\right\|+\cdots+\left\|\pi^{\ell}\right\|+2(\ell-1)}{2}}\right) .
$$

Asymptotically, expressions (54) and (55) differ by a multiplicative factor which concludes the proof of our claim.

It follows that Theorem 2.5 is applicable and $\omega_{\frac{1}{\sqrt{2 n_{k}}}} T_{\leq \alpha n_{k}}^{(k)}(x)$ indeed converges in probability to a limit shape $\Omega_{\alpha}$ which is uniquely determined by the sequence of its free cumulants

$$
R_{k}\left(\Omega_{\alpha}\right)=\alpha^{k-1} R_{k}\left(\Omega_{1}\right)
$$


The problem of finding the limit curves explicitly might be computationally challenging, see Sect. 7. Nevertheless in some cases we might be lucky to have a concrete final answer, see below.

\subsubsection{The example of Linusson, Potka and Sulzgruber}

Pittel and Romik [33] studied asymptotics of random standard Young tableaux having a square shape; in other words it was the non-shifted analogue of the problem studied by Linusson, Potka and Sulzgruber in [25]. The result of Pittel and Romik implies in particular that the (scaled down) level curves of such random standard Young tableaux converge in probability to a family of curves defined for $-1<z<1$ by

$$
\Omega_{\alpha}(z)=\sup \{x+y: 0 \leq x, y \leq 1 \text { and } x-y=z \text { and } L(x, y) \leq \alpha\}
$$

for some explicit function $L$ which describes the limit shape of the tableau.

On the other hand, the results of Biane [4, Theorem 1.5.1] also imply convergence of rescaled level curves to some family of curves $\Omega_{\alpha}$ which are defined via the free cumulants:

$$
R_{k}\left(\Omega_{\alpha}\right)=\alpha^{k-1} R_{k}\left(\Omega_{1}\right)
$$

where $\Omega_{1}$ describes the profile of the square Young diagram with a unit area.

By combining the results of Pittel and Romik with these of Biane it follows that both limits are equal. In particular, the curves (56) have known free cumulants (57).

The problem studied by Linusson, Potka and Sulzgruber [25] of asymptotics of random shifted staircase tableaux fits into the framework of Theorem 1.1 with the limit shape $\Omega_{1}$ the same as the limit shape in the problem of Pitman and Romik. Furthermore, in the proof of Theorem 1.1 we showed that the level curves $\Omega_{\alpha}$ fulfil the same Eq. (57). It follows that the level curves for the asymptotics of shifted staircase tableaux are the same as the limit level curves for square Young tableaux which was already observed in [25].

\section{How to recover the limit shape?}

The results of the current paper (such as Theorem 2.5) describe the limit shape of one or another class of large Young diagrams (this limit is given by some continual Young diagram $\omega: \mathbb{R} \rightarrow \mathbb{R}_{+}$) in terms of the sequence of its free cumulants $R_{2}(\omega), R_{3}(\omega), \ldots$ For Readers interested in concrete applications such a description might be not sufficient and they would prefer to recover the limit shape $\omega$ itself. This problem has a well-known answer; for Reader's convenience we shall review it briefly.

\subsection{Free cumulants, Cauchy transform, free probability}

In Sect. 2.6 we defined free cumulants $R_{2}, R_{3}, \ldots$ for a continual Young diagram $\omega$ quite directly via the functionals $S_{2}, S_{3}, \ldots$, cf. (21), which have a direct geometric 
interpretation in terms of the shape of $\omega$. The advantage of this approach lies in its simplicity.

Also, as we already discussed in the proof of Theorem 2.5, by inverting the relationship between $\left(R_{n}\right)$ and $\left(S_{n}\right)$, cf. (23), we see that the sequence of free cumulants determines uniquely the sequence of moments of the measure $\sigma_{\omega}$ considered in (20). Under some mild assumptions Hamburger moment problem [1] has a unique solution which shows that the measure $\sigma_{\omega}$ is uniquely determined. In the case when such an existential result is not enough and one would like to find the measure $\sigma_{\omega}$ explicitly, we will need some tools of the complex analysis. For this reason we need to recall the equivalent, original definition of free cumulants for Young diagrams. Originally, such free cumulants for Young diagrams were defined via a two-step process [4] which we recall in the following.

\subsubsection{The first step}

Kerov [21, Sect. 7] associated to a given continual Young diagram $\omega$ two measures on the real line. The first one, called Rayleigh measure, is a signed measure

$$
\tau=\tau_{\omega}=\frac{1}{2} \omega^{\prime \prime}
$$

given by the second derivative of $\omega$ in the distributional sense. The second one, called transition measure, is the unique probability measure $\mu=\mu_{\omega}$ for which the corresponding Cauchy-Stieltjes transform is given by

$$
G(z)=G_{\mu}(z)=\int_{\mathbb{R}} \frac{1}{z-u} \mu(\mathrm{d} u)=\exp \int_{\mathbb{R}} \log \frac{1}{z-u} \tau(\mathrm{d} u)
$$

for $z \in \mathbb{C} \backslash \mathbb{R}$. In our setup the transition measure is compactly supported.

\subsubsection{The second step}

Free cumulants originate in the context of the random matrix theory and Voiculescu's free probability theory, see [30] for an overview. For a compactly supported probability measure $\mu$ the corresponding $R$-transform is an analytic function $R=R_{\mu}$ on a neighbourhood of 0 on the complex plane which fulfils the equation

$$
G\left[R(z)+\frac{1}{z}\right]=z
$$

for $z \in \mathbb{C}$ in some neighbourhood of zero. In the specific setup which we consider in the current paper when $\mu=\mu_{\omega}$ is the transition measure of a continual Young diagram

$$
R(z)=\sum_{n \geq 2} R_{n} z^{n-1}
$$


turns out to be the generating function of the sequence of the free cumulants $R_{2}, R_{3}, \ldots$ presented in Sect. 2.6; the proof and additional context can be found in see [7, Sect. 3].

\subsection{How to recover the limit shape?}

Equality (58) implies that

$$
(-1) \frac{\mathrm{d}}{\mathrm{d} z} \log G(z)=\int_{\mathbb{R}} \frac{1}{z-u} \tau(\mathrm{d} u) ;
$$

in other words the left-hand side coincides with the Cauchy-Stieltjes transform of the signed measure $\tau$. The measure $\tau$ can be recovered from its Cauchy-Stieltjes tranform by Stieltjes inversion formula [30, Sect. 3.1] which in our context takes the form

$$
\begin{gathered}
\tau[(a, b)]+\frac{\tau(\{a, b\})}{2}=\frac{1}{\pi} \lim _{\epsilon \rightarrow 0^{+}} \int_{a}^{b} \Im \frac{\mathrm{d}}{\mathrm{d} z} \log G(z+i \epsilon) \\
=\frac{1}{\pi} \lim _{\epsilon \rightarrow 0^{+}}[\Im \log G(b+i \epsilon)-\Im \log G(a+i \epsilon)] \\
=\frac{1}{\pi} \lim _{\epsilon \rightarrow 0^{+}}[\arg G(b+i \epsilon)-\arg G(a+i \epsilon)] \in[0,1]
\end{gathered}
$$

for any real numbers $a<b$. Note that the left-hand side is equal to

$$
\frac{\omega^{\prime}(b)-\omega^{\prime}(a)}{2}
$$

for all $a<b$ for which the derivative of $\omega$ is well-defined.

Thus by considering the limit $a \rightarrow-\infty$ we get a formula for the cumulative distribution function of $\tau$ :

$$
\frac{\omega^{\prime}(z)+1}{2}=\tau[(-\infty, z)]=1+\frac{1}{\pi} \lim _{\epsilon \rightarrow 0^{+}} \arg G(z+i \epsilon) .
$$

which holds true for all $z \in \mathbb{R}$ for which $\omega^{\prime}(z)$ is well-defined. It follows that the shape of the continual Young diagram is given by

$$
\begin{aligned}
\omega(x)= & -x+2 \int_{-\infty}^{x}\left[1+\frac{1}{\pi} \lim _{\epsilon \rightarrow 0^{+}} \arg G(z+i \epsilon)\right] \mathrm{d} z \\
& =x-\frac{2}{\pi} \int_{x}^{\infty} \lim _{\epsilon \rightarrow 0^{+}} \arg G(z+i \epsilon) \mathrm{d} z .
\end{aligned}
$$

To summarize: in order to find the continual diagram $\omega$ it is enough to know its Cauchy transform $G$. For some classical concrete examples of finding the Cauchy transform when the sequence of free cumulants is known we refer to [30, Sect. 3.1]. In the following we will analyse in detail one concrete example. 


\subsection{Example: Schur-Weyl measure for $c=1$}

We will calculate explicitly the Schur-Weyl limit curve $\Omega_{c}^{\mathrm{SW}}$ (cf. Sect. 1.7.2) in the special case $c=1$. We look for a continual Young diagram with the sequence of free cumulants given by

$$
R_{k}= \begin{cases}\left(\frac{1}{\sqrt{2}}\right)^{k-2} & \text { if } k \text { is even } \\ 0 & \text { otherwise }\end{cases}
$$

This sequence of free cumulants was considered by Deya and Nourdin [8, Sect. 2.5]. With our notations the $R$-transform is given by

$$
R(z)=\frac{2 z}{2-z^{2}}
$$

note that [8] use a definition of $R$-transform which differs by a factor of $z$. Equation (59) shows therefore that $y=G(z)$ is a solution to the cubic equation

$$
z y^{3}+y^{2}-2 z y+2=0
$$

For $z \neq 0$ this equation has three solutions given by Cardan's formulae. Any Cauchy transform $G(z)$ fulfils some known properties (such as asymptotics for $z \rightarrow \infty$ ); by checking them Deya and Nourdin are able to pinpoint the right solution of the cubic equation which gives $G(z)$. Thus we have a closed formula for $G(z)$. Regretfully, computation of the argument $\arg G(z)$, even if $z$ converges to the real line, is a computational challenge; in particular we did not attempt to find a closed formula for the integrals appearing in (60). Even though we are not able to evaluate these integrals they are analytic functions. It follows that the limit curve $\Omega_{c}^{\mathrm{SW}}$ is piecewise analytic.

On the bright side, these integrals are easily accessible for a numerical integration and the limit curve $\Omega_{c}^{S W}$ can be efficiently calculated, see Fig. 5.

\subsection{Schur-Weyl measures, the generic case}

The above discussion of the special case $c=1$ is applicable also in the generic case. Note, however, that for some values of $c$ the behavior of the Cauchy transform and its singularities might be different.

For example, in the case $c=2$ shown on Fig. 6 the support of the Rayleigh measure is a union of two intervals (located symmetrically with respect to 0 ) and of the point $\{0\}$; in particular it follows that $\Omega_{2}^{\mathrm{SW}}(x)=|x|+\frac{\sqrt{2}}{2}$ for $x$ in a small neighbourhood of 0 .

Since this topic is vast and the current paper is already lengthy we postpone more details to a forthcoming publication.

Acknowledgements Research of SM was supported by JSPS KAKENHI Grant No. 17K05281. Research of PŚ was supported by Narodowe Centrum Nauki, Grant No. 2017/26/A/ST1/00189. 
Open Access This article is licensed under a Creative Commons Attribution 4.0 International License, which permits use, sharing, adaptation, distribution and reproduction in any medium or format, as long as you give appropriate credit to the original author(s) and the source, provide a link to the Creative Commons licence, and indicate if changes were made. The images or other third party material in this article are included in the article's Creative Commons licence, unless indicated otherwise in a credit line to the material. If material is not included in the article's Creative Commons licence and your intended use is not permitted by statutory regulation or exceeds the permitted use, you will need to obtain permission directly from the copyright holder. To view a copy of this licence, visit http://creativecommons.org/licenses/by/4.0/.

\section{Appendix A: Preliminaries on spin representation theory}

We reviewing the rudiments of the spin representation theory of the symmetric groups. For more details and bibliographic references we refer to [16,22,45,50].

\section{A.1. Linear and projective representations}

\section{A.1.1. Linear representations}

Recall that a linear representation of a finite group $G$ is a group homomorphism $\psi: G \rightarrow \mathrm{GL}(V)$ to the group of linear transformations $\mathrm{GL}(V)$ of some finitedimensional complex vector space $V$.

\section{A.1.2. Projective representations}

A projective representation of a finite group $G$ is a group homomorphism $\psi: G \rightarrow$ $\operatorname{PGL}(V)$ to the group of projective linear transformations $\operatorname{PGL}(V)=\mathrm{GL}(V) / \mathbb{C}^{\times}$ of the projective space $P(V)$ for some finite-dimensional complex vector space $V$. Equivalently, a projective representation can be viewed as a map $\phi: G \rightarrow \operatorname{GL}(V)$ to the general linear group with the property that

$$
\psi(x) \psi(y)=c_{x, y} \psi(x y)
$$

holds true for all $x, y \in G$ for some non-zero scalar $c_{x, y} \in \mathbb{C}^{\times}$.

Each irreducible linear representation $\psi: G \rightarrow \mathrm{GL}(V)$ gives rise to its projective version $\psi: G \rightarrow \operatorname{PGL}(V)$. The irreducible projective representations which cannot be obtained in this way are called irreducible spin representations and are in the focus of the current paper.

\section{A.2. Spin symmetric group and spin characters}

The spin group $\widetilde{\mathfrak{S}}_{n}$ [37] is a double cover of the symmetric group:

$$
1 \longrightarrow \mathbb{Z}_{2}=\{1, z\} \longrightarrow \widetilde{\mathfrak{S}}_{n} \longrightarrow \mathfrak{S}_{n} \longrightarrow 1 \text {. }
$$


More specifically, it is the group generated by $t_{1}, \ldots, t_{n-1}, z$ subject to the relations:

$$
\begin{aligned}
z^{2} & =1, & & \\
z t_{i} & =t_{i} z, & & \\
\left(t_{i} t_{i+1}\right)^{3} & =z & & \text { for } i \in[n-1], \\
t_{i} t_{j} & =z t_{j} t_{i} & & \text { for } i \in[n-2],
\end{aligned}
$$

we use the convention that $[k]=\{1, \ldots, k\}$. Under the mapping $\widetilde{\mathfrak{S}}_{n} \rightarrow \mathfrak{S}_{n}$ the generators $t_{1}, \ldots, t_{n-1}$ are mapped to the Coxeter tranpositions $(1,2),(2,3), \ldots,(n-$ $1, n) \in \mathfrak{S}_{n}$.

The main advantage of the spin group comes from the fact that any projective representation $\psi: \mathfrak{S}_{n} \rightarrow$ PGL( $\left.V\right)$ of the symmetric group can be lifted uniquely to a linear representation $\widetilde{\psi}: \widetilde{\mathfrak{S}}_{n} \rightarrow \mathrm{GL}(V)$ of the spin group so that the following diagram commutes:

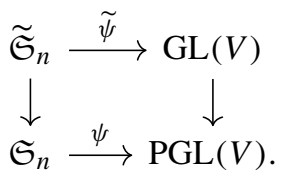

In this way the projective representation theory of the symmetric group $\mathfrak{S}_{n}$ is equivalent to the linear representation theory of the spin group $\widetilde{\mathfrak{S}}_{n}$ which allows to speak about the characters.

The irreducible spin representations of $\mathfrak{S}_{n}$ turn out to correspond to irreducible linear representations of the spin group algebra $\mathbb{C S}_{n}^{-}:=\mathbb{C}_{n} /\langle z+1\rangle$ which is the quotient of the group algebra $\mathbb{C} \widetilde{S}_{n}$ by the ideal generated by $(z+1)$. Equivalently $\mathbb{C S}_{n}^{-}=\langle 1-z\rangle \subset \mathbb{C}_{\mathfrak{S}_{n}}$ can be identified with the ideal generated by the projection $\frac{1-z}{2}$.

\section{A.3. Conjugacy classes of $\widetilde{\mathfrak{S}}_{n}$}

We denote by $\mathcal{O P}$ the set of odd partitions, i.e. partitions which consist only of odd parts and by $\mathcal{O P}$ the set of odd partitions of a given integer $n \geq 0$.

We denote by $\mathcal{S P}_{n}^{+}$(respectively, $\mathcal{S P}{ }_{n}^{-}$) the set of strict partitions $\xi \in \mathcal{S P}_{n}$ with the property that the length

$$
\|\xi\|:=|\xi|-\ell(\xi)
$$

is even (respectively, odd), see Fig. 9.

For a partition $\pi \vdash n$ we denote by $C_{\pi} \subset \widetilde{\mathfrak{S}}_{n}$ the set of elements of the spin group which are mapped-under the canonical homomorphism $\widetilde{\mathfrak{S}}_{n} \rightarrow \mathfrak{S}_{n}$ - to permutations with the cycle-type given by $\pi$.

Schur [37] proved the following dichotomy for $\pi \vdash n$ :

- if one of the following two conditions is fulfilled: 
- $\pi \in \mathcal{O P}_{n}$, or

- $\pi \in \mathcal{S P}_{n}^{-}$ then $C_{\pi}$ splits into a pair of conjugacy classes of $\widetilde{\mathfrak{S}}_{n}$ which will be denoted by $C_{\pi}^{ \pm}$

- otherwise, $C_{\pi}$ is a conjugacy class of $\widetilde{\mathfrak{S}}_{n}$.

\section{A.4. Conjugacy classes and spin characters}

Any spin character vanishes on the conjugacy class $C_{\pi}$ which does not split, cf. $[45$, p. 95]. For this reason, from the viewpoint of the spin character theory only the conjugacy classes $C_{\pi}^{ \pm}$are interesting.

Spin representations are exactly the ones which map the central element $z \in \widetilde{\mathfrak{S}}_{n}$ to $-\operatorname{Id} \in \operatorname{GL}(V)$. Since $C_{\pi}^{-}=z_{\pi} C_{\pi}^{+}$, it follows that the value of any spin character on $C_{\pi}^{-}$is the opposite of its value on $C_{\pi}^{+}$. For this reason, from the viewpoint of the spin character theory the conjugacy classes $C_{\pi}^{-}$are redundant and it is enough to consider the character values only on the conjugacy classes $C_{\pi}^{+}$.

From the viewpoint of the asymptotic representation theory it is natural to consider some sequence of groups together with some natural inclusions; in our case this is the sequence

$$
\widetilde{\mathfrak{S}}_{1} \subset \widetilde{\mathfrak{S}}_{2} \subset \widetilde{\mathfrak{S}}_{3} \subset \cdots
$$

of spin groups. Such a setup allows to relate a conjugacy class of a smaller group to some conjugacy class in the bigger group and, in this way, to evaluate the irreducible characters of the bigger group on the conjugacy classes of the smaller one.

Regretfully, the conjugacy classes $C_{\pi}^{+}$which correspond to $\pi \in \mathcal{S P}_{n}^{-}$do not behave nicely under such inclusions. Indeed, on the level of the symmetric groups the inclusion $\mathfrak{S}_{n} \subset \mathfrak{S}_{n+k}$ corresponds to adding $k$ fixpoints to a given permutation; in other words the set $C_{\pi} \subset \widetilde{\mathfrak{S}}_{n}$ corresponds to $C_{\pi, 1^{k}} \subset \widetilde{\mathfrak{S}}_{n+k}$ and the latter does not split because

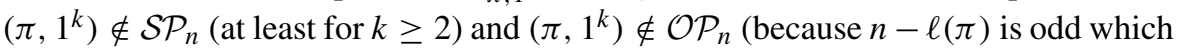
implies that at least one part of $\pi$ is even).

For this reason, for the purposes of the asymptotic representation theory it is enough to consider only the conjugacy classes $C_{\pi}^{+}$for $\pi \in \mathcal{O} \mathcal{P}_{n}$.

\section{A.5. Irreducible spin representations}

The relationship between strict partitions and the irreducible spin representations of the symmetric groups is not a bijective one. Nevertheless, as we shall discuss below, this non-bijectivity can be ignored to large extent.

More specifically (see [37, p. 235] and [45, Theorem 7.1]), each $\xi \in \mathcal{S P}_{n}^{+}$corresponds to a single irreducible representation. We denote by its character by $\phi^{\xi}$.

On the other hand, each $\xi \in \mathcal{S P}_{n}^{-}$corresponds to a pair of irreducible spin representations with equal dimensions; we denote their characters by $\phi_{+}^{\xi}$ and $\phi_{-}^{\xi}$. These two characters coincide on the conjugacy classes $C_{\pi}^{ \pm}$over $\pi \in \mathcal{O} \mathcal{P}_{n}$. For the purposes of the current paper we not need to evaluate the characters on $C_{\pi}^{ \pm}$for $\pi \in \mathcal{S P}_{n}^{-}$; for 
this reason we do not have to distinguish between $\phi_{+}^{\xi}$ and $\phi_{-}^{\xi}$ and we may denote them by the same symbol $\phi^{\xi}$.

\section{A.6. Superrepresentations}

In order to avoid the aforementioned difficulty related to the fact that the relationship between the irreducible representations of $\mathbb{C S}_{n}^{-}$and $\mathcal{S P}_{n}$ is not bijective we may change our setup to superalgebras and their superrepresentations. The following presentation is based on [22, Chapters 12, 13, 22].

We recall that a superalgebra is defined as an algebra $\mathcal{A}$ which is equipped with some $\mathbb{Z}_{2}$-grading $\mathcal{A}=\mathcal{A}_{\overline{0}} \oplus \mathcal{A}_{\overline{1}}$. Similarly, a superspace is a linear space $V$ equipped with some decomposition $V=V_{\overline{0}} \oplus V_{\overline{1}}$. The algebra $\operatorname{End}(V)$ of endomorphisms of a superspace carries a natural structure of a superalgebra by declaring that $X \in \operatorname{End}(V)$ is homogeneous of degree $i \in \mathbb{Z}_{2}=\{\overline{0}, \overline{1}\}$ if and only if for any homogeneous vector $v \in V_{j}$ with $j \in \mathbb{Z}_{2}$ we have that $X(v) \in V_{i+j}$.

A superrepresentation $\psi: \mathcal{A} \rightarrow \operatorname{End}(V)$ of a superalgebra is an algebra homomorphism which has the additional property that for any homogeneous element $x \in \mathcal{A}_{i}$ of degree $i \in \mathbb{Z}_{2}$ its image $\psi(x) \in \operatorname{End}(V)$ is also homogeneous of degree $i$.

We define a superalgebra structure on the spin group algebra $\mathbb{C} \widetilde{\mathfrak{S}}_{n}$ by declaring that the linear space of homogeneous elements of degree $\overline{0}$ (respectively, $\overline{1}$ ) is spanned by the group elements $g \in \widetilde{\mathfrak{S}}_{n}$ which under the canonical projection $\widetilde{\mathfrak{S}}_{n} \rightarrow \mathfrak{S}_{n}$ are mapped to even (respectively, odd) permutations. Then irreducible superrepresentations of $\mathbb{C S}_{n}^{-}$are in a canonical bijective correspondence with strict partitions in $\mathcal{S P}_{n}$ [22, Theorem 22.3.1].

[Here and in the following, the text in square brackets-such as this one-is intended only for Readers who are proficient in the terminology related to superalgebras. It provides some additional context but is not necessary for our purposes.]

We consider the irreducible superrepresentation which corresponds to $\xi \in \mathcal{S P}^{+}$. [It turns out to be of type M.] If we forget the superalgebra structure, it becomes an irreducible representation of the algebra $\mathbb{C S}_{n}^{-}$with the character $\phi^{\xi}$, as discussed in Sect. "Appendix A.5".

We consider now the irreducible superrepresentation which corresponds to $\xi \in$ $\mathcal{S P}^{-}$. [It turns out to be of type Q.] If we forget the superalgebra structure, it becomes a direct sum of the two irreducible representations of the algebra $\mathbb{C S}_{n}^{-}$with the characters $\phi_{ \pm}^{\xi}$, as discussed in Sect. "Appendix A.5". In particular, its character is equal to $\phi_{+}^{\xi}+\phi_{-}^{\xi}$.

\section{A.7. Spin characters: conclusion}

For each strict partition $\xi \in \mathcal{S} \mathcal{P}_{n}$ and each odd partition $\pi \in \mathcal{O P} \mathcal{P}_{n}$ the value of the irreducible spin character

$$
\phi^{\xi}(\pi)=\operatorname{Tr} \psi^{\xi}\left(c^{\pi}\right)
$$

is well defined, where $c^{\pi} \in C_{\pi}^{+}$is a representative of the of the conjugacy class $C_{\pi}^{+}$, cf. [45, Eq. (2.1)]. 


\section{References}

1. Akhiezer, N.I.: The Classical Moment Problem and Some Related Questions in Analysis. Translated by N. Kemmer. Hafner Publishing Co., New York, pp. X+253 (1965)

2. Bernstein, D., Henke, A., Regev, A.: Maximal projective degrees for strict partitions. Electron. J. Combin. 14(1), 59 (2007)

3. Biane, P.: Approximate factorization and concentration for characters of symmetric groups. Int. Math. Res. Not. 4, 179-192 (2001). https://doi.org/10.1155/S1073792801000113

4. Biane, P.: Representations of symmetric groups and free probability. Adv. Math. 138(1), 126-181 (1998). https://doi.org/10.1006/aima.1998.1745

5. Borodin, A.M.: Multiplicative central measures on the Schur graph. In: Zap. Nauchn. Sem. S.Peterburg. Otdel. Mat. Inst. Steklov. (POMI) 240.Teor. Predst. Din. Sist. Komb. i Algoritm. Metody. 2, pp. 44-52, 290-291 (1997). https://doi.org/10.1007/BF02175824

6. Brillinger, D.R.: The calculation of cumulants via conditioning. Ann. Inst. Stat. Math. 21(1), 215-218 (1969). https://doi.org/10.1007/BF02532246

7. Dołęga, M., Féray, V., Śniady, P.: Explicit combinatorial interpretation of Kerov character polynomials as numbers of permutation factorizations. Adv. Math. 225(1), 81-120 (2010). https://doi.org/10.1016/ j.aim.2010.02.011

8. Deya, A., Nourdin, I.: Convergence of Wigner integrals to the tetilla law. ALEA Lat. Am. J. Probab. Math. Stat. 9, 101-127 (2012)

9. Davis, R., Sagan, B.: Pattern-avoiding polytopes. Sém. Lothar. Combin. 78B, Art. 2, 12 (2017)

10. De Stavola, D.: Asymptotic results for representation theory (2017). arXiv:1805.04065 [math.CO]

11. Dołęga, M., Śniady, P.: Gaussian fluctuations of Jackdeformed random Young diagrams. Probab. Theory Relat. ields 174(1-2), 133-176 (2019). https://doi.org/10.1007/s00440-018-0854-9

12. Fishel, S., Nelson, L.: Chains of maximum length in the Tamari lattice. Proc. Am. Math. Soc. 142(10), 3343-3353 (2014). https://doi.org/10.1090/S0002-9939-2014-12069-7

13. Hoffman, P.N., Humphreys, J.F.: Projective Representations of the Symmetric Groups. Oxford Mathematical Monographs. Q-functions and Shifted Tableaux, p. xiv+304. Oxford Science Publications, New York (1992)

14. Hora, A.: The Limit Shape Problem for Ensembles of Young Diagrams. SpringerBriefs in Mathematical Physics, vol. 17, p. ix+73. Springer, Tokyo (2016). https://doi.org/10.1007/978-4-431-56487-4

15. Ivanov, V., Olshanski, G.: Kerov's central limit theorem for the Plancherel measure on Young diagrams. In: Symmetric functions 2001: surveys of developments and perspectives, vol. 74. NATO Sci. Ser. II Math. Phys. Chem. Kluwer Acad. Publ., Dordrecht, pp. 93-151 (2002). https://doi.org/10.1007/97894-010-0524-1_3

16. Ivanov, V.N.: Gaussian limit for projective characters of large symmetric groups. J. Math. Sci. 121(3), 2330-2344 (2004). https://doi.org/10.1023/B:JOTH.0000024615.07311.fe

17. Ivanov, V.: Plancherel measure on shifted Young diagrams. In: Representation Theory, Dynamical Systems, and Asymptotic Combinatorics, vol. 217. Amer. Math. Soc. Transl. Ser. 2. Amer. Math. Soc., Providence, RI, pp. 73-86 (2006). https://doi.org/10.1090/trans2/217/06

18. Ivanov, V.N.: Dimensions of skew-shifted young diagrams and projective characters of the infinite symmetric group. J. Math. Sci. 96(5), 3517-3530 (1999). https://doi.org/10.1007/BF02175830

19. Kerov, S.V.: Transition probabilities of continual Young diagrams and the Markov moment problem. Funkt. Anal. Prilozhen. 27(2), 32-49, 96 (1993). https://doi.org/10.1007/BF01085981

20. Kerov, S.: Gaussian limit for the Plancherel measure of the symmetric group. C. R. Acad. Sci. Paris Sér. I Math. 316(4), 303-308 (1993)

21. Kerov, S.: Interlacing measures. In: Kirillov's Seminar on Representation Theory, vol. 181. Amer. Math. Soc. Transl. Ser. 2. Amer. Math. Soc., Providence, RI, pp. 35-83 (1998). https://doi.org/10. 1090/trans2/181/02

22. Kleshchev, A.: Linear and Projective Representations of Symmetric Groups. Cambridge Tracts in Mathematics, vol. 163, p. xiv+277. Cambridge University Press, Cambridge (2005). https://doi.org/ 10.1017/CBO9780511542800

23. Kerov, S., Olshanski, G.: Polynomial functions on the set of Young diagrams. C. R. Acad. Sci. Paris Sér. I Math. 319.2, 121-126 (1994)

24. Linusson, S., Potka, S.: New properties of the Edelman-Greene bijection (2018). arXiv:1804.10034 [math.CO] 
25. Linusson, S., Potka, S., Sulzberger, R.: On random shifted standard Young tableaux and 132-avoiding sorting networks (2018). arXiv:1804.01795 [math.CO]

26. Logan, B.F., Shepp, L.A.: A variational problem for random Young tableaux. Adv. Math. 26(2), 206222 (1977). https://doi.org/10.1016/0001-8708(77)90030-5

27. Macdonald, I.G.: Symmetric Functions and Hall Polynomials. Oxford Mathematical Monographs. With contributions by A. Zelevinsky, Second edn, p. x+475. Oxford Science Publications, New York (1995)

28. Matsumoto, S.: A spin analogue of Kerov polynomials. In: Symmetry, Integrability and Geometry: Methods and Applications (SIGMA), vol. 14 (2018), p. 053. eprint: 1803.01121

29. Méliot, P.-L.: Representation Theory of Symmetric Groups. Discrete Mathematics and its Applications (Boca Raton), p. xvi+666. CRC Press, Boca Raton (2017). https://doi.org/10.1201/9781315371016

30. Mingo, J.A., Speicher, R.: Free Probability and Random Matrices. Fields Institute Monographs. Fields Institute for Research in Mathematical Sciences, vol. 35, p. xiv+336. Springer, New York (2017). https://doi.org/10.1007/978-1-4939-6942-5

31. Matsumoto, S., Śniady, P.: Linear versus spin: representation theory of the symmetric groups. To appear in Algebraic combinatorics (2018). arXiv:1811.10434 [math.CO]

32. Okounkov, A.: The uses of random partitions. In: Fourteenth International Congress on Mathematical Physics. Word Scientists, pp. 379-403 (2003)

33. Pittel, B., Romik, D.: Limit shapes for random square Young tableaux. Adv. Appl. Math. 38(2), 164-209 (2007). https://doi.org/10.1016/j.aam.2005.12.005

34. Proctor, R.A.: Dynkin diagram classification of $\lambda$-minuscule Bruhat lattices and of d-complete posets. J. Algebr. Combin. 9(1), 61-94 (1999). https://doi.org/10.1023/A:1018615115006

35. Sagan, B.: On selecting a random shifted Young tableau. J. Algorithms 1(3), 213-234 (1980). https:// doi.org/10.1016/0196-6774(80)90010-3

36. Sagan, B.E.: Shifted tableaux, Schur Q-functions, and a conjecture of R. Stanley. J. Combin. Theory Ser. A 45.1, 62-103 (1987). https://doi.org/10.1016/0097-3165(87)90047-1

37. Schur, J.: Über die Darstellung der symmetrischen und der alternierenden Gruppe durch gebrochene lineare Substitutionen. J. Reine Angew. Math. 139, 155-250 (1911). https://doi.org/10.1515/crll.1911. 139.155

38. Schilling, A., et al.: Braid moves in commutation classes of the symmetric group. Eur. J. Combin 62, 15-34 (2017). https://doi.org/10.1016/j.ejc.2016.10.008

39. Serrano, L.: The shifted plactic monoid. Math. Z. 266(2), 363-392 (2010). https://doi.org/10.1007/ s00209-009-0573-0

40. Śniady, P.: Asymptotics of symmetric groups representations. Introduction to the habilitation thesis. University of Wrocław (2006)

41. Sniady, P.: Gaussian fluctuations of characters of symmetric groups and of Young diagrams. Probab. Theory Relat. Fields 136(2), 263-297 (2006). https://doi.org/10.1007/s00440-005-0483-y

42. Śniady, P.: Structure coefficients for Jack characters: approximate factorization property (2016). arXiv:1603.04268v1 [math.CO]

43. Śniady, P.: Asymptotics of Jack characters. J. Combin. Theory Ser. A 166, 91-143 (2019). https://doi. org/10.1016/j.jcta.2019.02.020

44. Speed, T.P.: Cumulants and partition lattices. Aust. J. Statist. 25(2), 378-388 (1983)

45. Stembridge, J.R.: Shifted tableaux and the projective representations of symmetric groups. Adv. Math. 74(1), 87-134 (1989). https://doi.org/10.1016/0001-8708(89)90005-4

46. Stembridge, J.R.: On the fully commutative elements of Coxeter groups. J. Algebr. Combin. 5(4), 353-385 (1996). https://doi.org/10.1023/A:1022452717148

47. Vershik, A.M.: Asymptotic combinatorics and algebraic analysis. In: Proceedings of the International Congress of Mathematicians, vol. 1, 2 (Zürich, 1994). Birkhäuser, Basel, pp. 1384-1394 (1995)

48. Veršik, A.M., Kerov, S.V.: Asymptotic behavior of the Plancherel measure of the symmetric group and the limit form of Young tableaux. Dokl. Akad. Nauk SSSR 233(6), 1024-1027 (1977)

49. Worley, D.R.: A theory of shifted Young tableaux. Thesis (Ph.D.)-Massachusetts Institute of Technology. ProQuest LLC, Ann Arbor, MI (1984)

50. Wan, J., Wang, W.: Lectures on spin representation theory of symmetric groups. In: Bull. Inst. Math. Acad. Sin. (N.S.) 7.1, pp. 91-164 (2012)

Publisher's Note Springer Nature remains neutral with regard to jurisdictional claims in published maps and institutional affiliations. 